\title{
Lines on Smooth Polarized K3-Surfaces
}

\section{Alex Degtyarev ${ }^{1}(\mathbb{D}$}

Received: 28 August 2017 / Revised: 29 August 2018 / Accepted: 28 September 2018 / Published online: 1 November 2018

(c) Springer Science+Business Media, LLC, part of Springer Nature 2018

\section{Abstract}

For each integer $D \geqslant 3$, we give a sharp bound on the number of lines contained in a smooth complex $2 D$-polarized $K 3$-surface in $\mathbb{P}^{D+1}$. In the two most interesting cases of sextics in $\mathbb{P}^{4}$ and octics in $\mathbb{P}^{5}$, the bounds are 42 and 36 , respectively, as conjectured in an earlier paper.

Keywords $K 3$-Surface - Sextic surface - Octic surface - Triquadric - Elliptic pencil · Integral lattice $\cdot$ Discriminant form

Mathematics Subject Classification Primary 14J28; Secondary 14J27 · 14N25

\section{Introduction}

All algebraic varieties considered in the paper are over $\mathbb{C}$.

\subsection{The Line Counting Problem}

The paper deals with a very classical algebra-geometric problem, viz. counting straight lines in a projective surface. We confine ourselves to the smooth $2 D$-polarized $K 3$ surfaces $X \subset \mathbb{P}^{D+1}, D \geqslant 3$, and obtain sharp upper bounds on the number of lines. Our primary interest are sextics in $\mathbb{P}^{4}(D=3)$ and octics in $\mathbb{P}^{5}(D=4)$; however, the same approach gives us a complete answer for all higher degrees/dimensions as well.

Recall (see [14]) that a projectively normal smooth $K 3$-surface $X \subset \mathbb{P}^{D+1}$ has projective degree $2 D$. Given a smooth embedding $\varphi: X \hookrightarrow \mathbb{P}^{D+1}$, the polarization

Editor in Charge: János Pach

The author was partially supported by the TÜBITTAK Grant $116 \mathrm{~F} 211$.

Alex Degtyarev

degt@fen.bilkent.edu.tr

1 Department of Mathematics, Bilkent University, 06800 Ankara, Turkey 
is the pull-back $\varphi^{*} \mathcal{O}_{\mathbb{P}^{D+1}}(1)$ regarded as a class $h \in H_{2}(X ; \mathbb{Z})$; one has $h^{2}=2 D$. Since each line $l \subset X$ is a (-2)-curve, it is uniquely determined by its homology class $[l] \in N S(X) \subset H_{2}(X ; \mathbb{Z})$; to simplify the notation, we identify lines and their classes. In particular, the set of lines is finite; its dual incidence graph Fn $X$ is called the Fano graph, and the primitive sublattice $\mathcal{F}_{h}(X) \subset H_{2}(X ; \mathbb{Z})$ generated over $\mathbb{Q}$ by $h$ and all lines $l \in \mathrm{Fn} X$ is called the Fano configuration of $X$. This polarized lattice, subject to certain restrictions (see Theorem 2.2), defines an equilinear family of $2 D$-polarized $K 3$-surfaces $X \subset \mathbb{P}^{D+1}$; on the other hand, the lattice $\mathcal{F}_{h}(X)$ is usually recovered from the graph Fn $X$ (see Sect. 2.4).

The case $D=2$, i.e., that of spatial quartics, is very classical and well known. The quartic $X_{64}$ maximizing the number of lines was constructed by Schur [15] as early as in 1882 . The problem kept reappearing here and there ever since, but no significant progress had been made until 1943, when Segre [16] published a paper asserting that 64 is indeed the maximal number of lines in a smooth spatial quartic. Recently, Rams and Schütt [12] discovered a gap in Segre's argument (but not the statement); they patched the proof and extended it to algebraically closed fields of all characteristics other than 2 or 3. Finally, in [5], we gave an alternative proof and a number of refinements of the statement, including the complete classification of all quartics carrying more than 52 lines. In particular, Schur's quartic is the only one with the maximal number 64 of lines.

All extremal (carrying more than 52 lines) quartics found in [5] are projectively rigid, as they are the so-called singular K3-surfaces. Recall that a $K 3$-surface $X$ is singular if its Picard rank is maximal, $\rho(X):=\operatorname{rk} N S(X)=20$. (This unfortunate term is not to be confused with singular $v s$. smooth projective models of surfaces.) Up to isomorphism, an abstract singular $K 3$-surface is determined by the oriented isomorphism type of its transcendental lattice

$$
T:=N S(X)^{\perp} \subset H_{2}(X ; \mathbb{Z}),
$$

which is a positive definite even integral lattice of rank 2 (see Sect. 2.1; the orientation is given by the class $[\omega] \in T \otimes \mathbb{C}$ of a holomorphic 2 -form on $X$ ); we use the notation $X(T)$, see Sect. 1.2. As a follow-up to [5] (and also motivated by [17]), in [3] I tried to study smooth projective models of singular $K 3$-surfaces $X(T)$ of small discriminant $\operatorname{det} T$. Unexpectedly, it was discovered that Schur's quartic $X_{64}$ can alternatively be characterized as the only smooth spatial model minimizing this discriminant: one has $X_{64} \cong X([8,4,8])$ (see Sect. 1.2 for the notation) and $\operatorname{det} T \geqslant 55$ for any other smooth quartic $X(T) \subset \mathbb{P}^{3}$.

After quartics, next most popular projective models of $K 3$-surfaces are sextics $X \subset \mathbb{P}^{4}$ and octics $X \subset \mathbb{P}^{5}$, and the results of [3] extend to these two classes: if a singular $K 3$-surface $X(T)$ admits a smooth sextic or octic model, then $\operatorname{det} T \geqslant 39$ or 32, respectively. In view of the alternative characterization of Schur's quartic $X_{64}$ discovered in [3], this classification, followed by a study of the models, suggested a conjecture that a smooth sextic $X \subset \mathbb{P}^{4}$ (respectively, octic $X \subset \mathbb{P}^{5}$ ) may have at most 42 (respectively, 36) lines. This conjecture is proved in the present paper (the cases $D=3,4$ in Theorem 1.1), even though the original motivating observation that 
discriminant minimizing singular $K 3$-surfaces maximize the number of lines fails already for degree 10 surfaces $X \subset \mathbb{P}^{6}$ (see Theorem 1.7).

Each sextic in $\mathbb{P}^{4}$ is a regular complete intersection of a quadric and a cubic. I am not aware of any previously known interesting examples of large configurations of lines in such surfaces. The maximal number 42 of lines is attained at a 1-parameter family containing the discriminant minimizing surfaces $X([2,1,20])$ and $X([6,3,8])$.

Most octics in $\mathbb{P}^{5}$ are also regular complete intersections: they are the so-called triquadrics, i.e., intersections of three quadrics. The most well-known example is the Kummer family

$$
\sum_{i=0}^{5} z_{i}=\sum_{i=0}^{5} a_{i} z_{i}=\sum_{i=0}^{5} a_{i}^{2} z_{i}=0, \quad a_{i} \in \mathbb{C}
$$

whose generic members contain 32 lines: famous Kummer's $16_{6}$ configuration (see, e.g., Dolgachev [6]). There are four other, less symmetric, configurations of 32 lines, either rigid or realized by 2-parameter families. However, 32 is not the maximum: there also are configurations with 33, 34, and 36 lines (see Table 1).

The space of octics contains a divisor composed by surfaces that need one cubic defining equation (see [14] and Sect. 2.2). We call these octics special and show that they do stand out in what concerns the line counting problem. Large Fano graphs of special octics ( $v s$. triquadrics) are described by Theorem 1.3.

\subsection{Common Notation}

We use the following notation for particular integral lattices of small rank (see Sect. 2.1):

- $\mathbf{A}_{p}, p \geqslant 1, \mathbf{D}_{q}, q \geqslant 4, \mathbf{E}_{6}, \mathbf{E}_{7}, \mathbf{E}_{8}$ are the positive definite root lattices generated by the indecomposable root systems of the same name (see [1]);

- $[a]:=\mathbb{Z} u, u^{2}=a$, is a lattice of rank 1 ;

- $[a, b, c]:=\mathbb{Z} u+\mathbb{Z} v, u^{2}=a, u \cdot v=b, v^{2}=c$, is a lattice of rank 2; when it is positive definite, we assume that $0<a \leqslant c$ and $0 \leqslant 2 b \leqslant a$ : then, $u$ is a shortest vector, $v$ is a next shortest one, and the triple $(a, b, c)$ is unique;

- $\mathbf{U}:=[0,1,0]$ is the hyperbolic plane.

Besides, $L(n), n \in \mathbb{Z}$, is the lattice obtained by the scaling of a given lattice $L$, i.e., multiplying all values of the form by a fixed integer $n \neq 0$.

To simplify the statements, we let the girth of a forest equal to infinity, so that the inequality girth $(\Gamma) \geqslant m$ means that $\Gamma$ has no cycles of length less than $m$.

When describing lists of integers, $a . . b$ means the full range $\mathbb{Z} \cap[a, b]$.

We denote by $\{a \bmod d\}$ the arithmetic progression $a+n d, \ldots, n \geqslant 0$, and use the shortcut $\left\{a_{1}, \ldots, a_{r} \bmod d\right\}:=\bigcup_{i}\left\{a_{i} \bmod d\right\}$ for finite unions. 


\subsection{Principal Results}

Given a field $K \subset \mathbb{C}$ and an integer $D \geqslant 2$, let $M_{K}(D)$ be the maximal number of lines defined over $K$ that a smooth $2 D$-polarized $K 3$-surface $X \subset \mathbb{P}^{D+1}$ defined over $K$ may have.

Most principal results of the paper are collected in Table 1; precise statements are found in the several theorems below. (For the sake of completeness, we also cite some results of [5] concerning quartics, i.e., $D=2$.) In the table, we list:

- the degrees $h^{2}=2 D$ for which extra information is available (marking with a ${ }^{\dagger}$ the two values that are special in the sense of Theorem 1.7),

- the bounds $M:=M(D)$ and $\bar{M}:=\bar{M}(D)$ used in Theorem 1.1 (according to which, $M(D)=M_{\mathbb{C}}(D)$ for all values of $D$ in the table), and

- the maximal number $M_{\mathbb{R}}:=M_{\mathbb{R}}(D)$ of real lines (see Theorem 1.4).

Then, for each value of $D$, we list, line-by-line, all Fano graphs $\Gamma:=$ Fn $X$ containing more than $\bar{M}(D)$ lines (the notation is explained below), marking with a $\ddagger$ those realized by real lines in real surfaces (see Theorem 1.4) and indicating

- the order $\mid$ Aut $\Gamma \mid$ of the full automorphism group of $\Gamma$ and

- the transcendental lattices $T:=\mathcal{F}_{h}(\Gamma)^{\perp}=N S(X)^{\perp} \in H_{2}(X ; \mathbb{Z})$ of generic smooth $2 D$-polarized $K 3$-surfaces $X$ with Fn $X \cong \Gamma$ (marking with a * the lattices resulting in pairs of complex conjugate equilinear families).

For the rigid configurations ( $\operatorname{rk} T=2$ ), we list, in addition,

- the determinant det $T$ (underlining the minimal ones, see Theorem 1.7),

- the numbers $(r, c)$ of, respectively, real and pairs of complex conjugate projective isomorphism classes of surfaces $X$ with Fn $X \cong \Gamma$, and

- the order $\mid$ Aut $X \mid$ of the group of projective automorphisms of $X$.

Each of the few non-rigid configurations $\Gamma$ appearing in the table is realized by a single connected equilinear deformation family $\mathcal{M}_{D}(\Gamma)$; we indicate the dimension $\operatorname{dim}\left(\mathcal{M}_{D}(\Gamma) / P G L(D+2, \mathbb{C})\right)=\operatorname{rk} T-2$ and, when known, the minimum of the discriminants det $T$ of the singular $K 3$-surfaces $X(T) \in \mathcal{M}_{D}(\Gamma)$.

If $D=2$, we use the notation for the extremal configurations introduced in [5]; otherwise, we refer to the isomorphism classes of the Fano graphs introduced and discussed in more details elsewhere in the paper. In both cases, the subscript is the number of lines in the configuration. For technical reasons, we subdivide Fano graphs into several classes (see Sect. 4.1) and study them separately, obtaining more refined bounds for each class. Thus, a Fano graph $\Gamma:=$ Fn $X$ and the configuration $\mathcal{F}_{h}(\Gamma):=$ $\mathcal{F}_{h}(X)$ are called

- triangular (the $\Psi_{*}$-series, see Theorem 8.1), if girth $(\Gamma)=3$; all extremal quartics also fall into this class,

- quadrangular (the $\Theta_{*}$-series, see Theorem 7.1), if $\operatorname{girth}(\Gamma)=4$,

- pentagonal (the $\Phi_{*}$-series, see Theorem 6.1), if girth $(\Gamma)=5$,

- astral (the $\Delta_{*}$-series, see Theorem 5.1), if $\operatorname{girth}(\Gamma) \geqslant 6$ and $\Gamma$ has a vertex $v$ of valency val $v \geqslant 4$. 
Table 1 Exceptional configurations (see Theorem 1.1)

\begin{tabular}{|c|c|c|c|c|c|c|c|c|}
\hline$D$ & $M, \bar{M}$ & $M_{\mathbb{R}}$ & $\Gamma$ & $\mid$ Aut $\Gamma \mid$ & det & $(r, c)$ & $\mid$ Aut $X \mid$ & $T:=\mathcal{F}_{h}(\Gamma)^{\perp}$ \\
\hline \multirow[t]{8}{*}{2} & \multirow[t]{8}{*}{64,52} & \multirow[t]{8}{*}{56} & $\mathbf{X}_{64}$ & 4608 & $\underline{48}$ & $(1,0)$ & 1152 & {$[8,4,8]$} \\
\hline & & & $\mathbf{X}_{60}^{\prime}$ & 480 & 60 & $(1,0)$ & 120 & {$[4,2,16]$} \\
\hline & & & $\mathbf{X}_{60}^{\prime \prime}$ & 240 & 55 & $(0,1)$ & 120 & {$[4,1,14]^{*}$} \\
\hline & & & $\mathbf{X}_{56}$ & 128 & 64 & $(0,1)$ & 32 & {$[8,0,8]^{*}$} \\
\hline & & & $\mathbf{Y}_{56^{\ddagger}}^{\ddagger}$ & 64 & 64 & $(1,0)$ & 32 & {$[2,0,32]$} \\
\hline & & & $\mathbf{Q}_{56}$ & 384 & 60 & $(1,0)$ & 96 & {$[4,2,16]$} \\
\hline & & & $\mathbf{X}_{54}$ & 384 & 96 & $(1,0)$ & 48 & {$[4,0,24]$} \\
\hline & & & $\mathbf{Q}_{54}$ & 48 & 76 & $(1,0)$ & 8 & {$[4,2,20]$} \\
\hline \multirow[t]{2}{*}{3} & \multirow[t]{2}{*}{42,36} & \multirow[t]{2}{*}{42} & $\Psi_{42}$ & 432 & 39 & \multicolumn{2}{|l|}{$\operatorname{dim}=1$} & $\mathbf{A}_{2} \oplus[-18]$ \\
\hline & & & $\Psi_{38}$ & 32 & 48 & \multicolumn{2}{|l|}{$\operatorname{dim}=1$} & $\mathbf{A}_{2} \oplus[-24]$ \\
\hline \multirow[t]{10}{*}{4} & \multirow[t]{10}{*}{36,30} & \multirow[t]{10}{*}{34} & $\Theta_{36}^{\prime}$ & 64 & $\underline{32}$ & $(1,0)$ & 16 & {$[4,0,8]$} \\
\hline & & & $\Theta_{36}^{\prime \prime}$ & 576 & $\overline{36}$ & $(1,0)$ & 144 & {$[6,0,6]$} \\
\hline & & & $\Theta_{34}^{\prime}{ }^{\ddagger}$ & 96 & & \multicolumn{2}{|l|}{$\operatorname{dim}=1$} & $\mathbf{U}(2) \oplus[12]$ \\
\hline & & & $\Theta_{33}$ & 192 & 80 & $(1,0)$ & 24 & {$[8,4,12]$} \\
\hline & & & $\Psi_{33}$ & 6912 & 36 & \multicolumn{2}{|l|}{$\operatorname{dim}=2$} & $\mathbf{U}^{2}(3)$ \\
\hline & & & $\Theta_{32}$ & 96 & 60 & $(1,0)$ & 24 & {$[4,2,16]$} \\
\hline & & & $\Theta_{32}^{\prime}{ }^{\ddagger}$ & 384 & & \multicolumn{2}{|l|}{$\operatorname{dim}=2$} & $\mathbf{U}(2) \oplus[-4] \oplus[4]$ \\
\hline & & & $\Theta_{32}^{\prime \prime}$ & 512 & & \multicolumn{2}{|l|}{$\operatorname{dim}=2$} & $\mathbf{U}(2) \oplus \mathbf{U}(4)$ \\
\hline & & & $\Theta_{32}^{\prime \prime \prime}$ & 768 & 36 & \multicolumn{2}{|l|}{$\operatorname{dim}=2$} & $\mathbf{U}^{2}(3)$ \\
\hline & & & $\Theta_{32}^{\mathrm{K}}$ * & 23040 & $\underline{32}$ & \multicolumn{2}{|l|}{$\operatorname{dim}=3$} & $\mathbf{U}^{2}(2) \oplus[-4]$ \\
\hline \multirow[t]{3}{*}{$5^{\dagger}$} & \multirow[t]{3}{*}{30,28} & \multirow[t]{3}{*}{28} & $\Phi_{30}^{\prime}$ & 240 & 100 & $(1,0)$ & 60 & {$[10,0,10]$} \\
\hline & & & $\Phi_{30}^{\prime \prime}$ & 40 & 75 & $(1,0)$ & 10 & {$[10,5,10]$} \\
\hline & & & $\Phi_{30}^{\prime \prime \prime}$ & 24 & 36 & $(1,0)$ & 12 & {$[4,2,10]$} \\
\hline \multirow[t]{2}{*}{6} & \multirow[t]{2}{*}{36,28} & \multirow[t]{2}{*}{36} & $\Phi_{30}^{\prime}$ & 240 & 60 & $(1,0)$ & 60 & {$[8,2,8]$} \\
\hline & & & $\Phi_{36}^{\prime \prime}$ & 1440 & $\underline{15}$ & $(1,0)$ & 720 & {$[2,1,8]$} \\
\hline \multirow[t]{2}{*}{7} & \multirow[t]{2}{*}{30,26} & \multirow[t]{2}{*}{26} & $\Phi_{30}^{\prime}$ & 240 & $\underline{20}$ & $(1,0)$ & 120 & {$[4,2,6]$} \\
\hline & & & $\Delta_{27}^{\prime \prime}$ & 72 & 99 & $(1,0)$ & 18 & {$[6,3,18]$} \\
\hline 8 & 32,24 & 24 & $\Delta_{32}^{\prime}$ & 2304 & 12 & $(1,0)$ & 1152 & {$[4,2,4]$} \\
\hline & & & $\Delta_{26}$ & 24 & 60 & $(1,0)$ & 6 & {$[4,2,16]$} \\
\hline & & & $\Delta_{25}^{\prime \prime}$ & 16 & 60 & $(1,0)$ & 4 & {$[4,2,16]$} \\
\hline & & & $\Phi_{25}$ & 80 & & $\operatorname{dim}=1$ & & $\mathbf{U}(5) \oplus[4]$ \\
\hline 9 & 25,24 & 25 & $\Phi_{25}$ & 80 & $\underline{15}$ & $\operatorname{dim}=1$ & & $\mathbf{U}(5) \oplus[2]$ \\
\hline $10^{\dagger}$ & 25,24 & 25 & $\Delta_{25}^{\prime}$ & 24 & $\overline{96}$ & $(0,1)$ & 6 & {$[4,0,24]$} \\
\hline & & & $\Phi_{25}$ & 80 & & $\operatorname{dim}=2$ & & $\mathbf{U}(5) \oplus \mathbf{U}$ \\
\hline & & & $\Lambda_{25}$ & 144 & 140 & $(1,0)$ & 12 & {$[12,2,12]$} \\
\hline 14 & 28,24 & 28 & $\Lambda_{28}$ & 336 & 7 & $(1,0)$ & 336 & {$[2,1,4]$} \\
\hline
\end{tabular}


All other graphs are locally elliptic (the $\Lambda_{*}$-series, see Sect. 4.2), i.e., one has val $v \leqslant 3$ for each vertex $v \in \Gamma$.

In our notation for particular graphs/configurations, the subscript always stands for the number of vertices/lines. The precise description of all graphs "named" in the paper is available electronically (in the form of GRAPE records) in [4]; in most cases, the implicit reference to [4] is, in fact, the definition of the graph.

\subsection{The Bounds}

Geometrically, apart from the spatial quartics, the two most interesting projective models of $K 3$-surfaces are sextics in $\mathbb{P}^{4}$ and octics (especially triquadrics) in $\mathbb{P}^{5}$. However, it turns out that the structure of the Fano graphs simplifies dramatically when the degree $h^{2}=2 D$ grows, and one can easily obtain the sharp bounds $M_{\mathbb{C}}(D)$ and $M_{\mathbb{R}}(D)$ for all values of $D$. Below, these bounds are stated for $D$ small and $D \rightarrow \infty$; the rather erratic precise values of

$$
21 \leqslant M_{\mathbb{C}}(D) \leqslant 24, D>20 \text { and } 19 \leqslant M_{\mathbb{R}}(D) \leqslant 24, D>16
$$

are postponed till Sect. 4.2 (see Corollaries 4.8 and 4.9, respectively).

For the next theorem, given a degree $h^{2}=2 D$, let $M:=M(D)$ and $\bar{M}:=\bar{M}(D)$ be as in Table 1 or $M(D)=\bar{M}(D)=24$ if $D$ is not found in the table. As in [5], in addition to the upper bound $|\mathrm{Fn} X| \leqslant M(D)$, we give a complete classification of all large (close to maximal) configurations of lines.

Theorem 1.1 (see Sect. 8.4) Let $X \subset \mathbb{P}^{D+1}$ be a smooth 2D-polarized K3-surface. Then one has $|\mathrm{Fn} X| \leqslant M(D)$. More precisely, $|\mathrm{Fn} X| \leqslant \bar{M}(D)$ unless $\Gamma:=\operatorname{Fn} X$ is one of the exceptional graphs (configurations) listed in Table 1.

Both bounds are sharp whenever $2 \leqslant D \leqslant 20$ or $D \in\{1,2,4,6,7,10 \bmod 12\}$; in particular, one has $M_{\mathbb{C}}(D)=M(D)$ for these values.

Addendum 1.2 (see Sect. 8.4) The complete set $\{|\mathrm{Fn} X|\}$ of values taken by the line count of a smooth $2 D$-polarized K3-surface $X \subset \mathbb{P}^{D+1}$ is as follows:

- if $D=2$, then $\{|\mathrm{Fn} X|\}=\{0$.. 52, 54, 56, 60, 64\} (see [5]);

- if $D=3$, then $\{|\mathrm{Fn} X|\}=\{0 \ldots 36,38,42\}$;

- if $D=4$, then $\{|\mathrm{Fn} X|\}=\{0$.. 30, 32, 33, 34, 36 $\}$.

As mentioned at the end of Sect. 1.1, the Fano graphs of special octics differ from those of triquadrics. They are described by the following theorem, which implies, in particular, that the graphs $\Theta_{*}^{*}$ in Table 1 are realized only by triquadrics, whereas $\Psi_{33}$ is realized only by special octics.

Theorem 1.3 (see Sect. 5.4) Let $X \subset \mathbb{P}^{5}$ be a smooth special octic, and assume that $|\mathrm{Fn} X| \geqslant 20$. Then one of the following statements holds:

(1) girth $(\mathrm{Fn} X)=3$ and $\mathrm{Fn} X \cong \Psi_{33}, \Psi_{29}, \Psi_{27}^{\prime}, \Psi_{27}^{\prime \prime}$ or $|\mathrm{Fn} X| \leqslant 25$, or

(2) $\operatorname{girth}(\operatorname{Fn} X)=4$ and $\operatorname{Fn} X \cong \Theta_{21}$ has a biquadrangle (see Definition 5.3). 
Conversely, if $X \subset \mathbb{P}^{5}$ is a smooth octic such that either $\operatorname{girth}(\operatorname{Fn} X)=3$ or $\operatorname{Fn} X$ has a biquadrangle, then $X$ is special.

The threshold $|\mathrm{Fn} X| \geqslant 20$ in Theorem 1.3 is optimal: there are graphs with 19 lines realized by both triquadrics and special octics (see Proposition 5.9).

For completeness, in Sect. 5.5 we discuss also "lines" in the hyperelliptic models

$$
X \rightarrow \Sigma_{D-2} \hookrightarrow \mathbb{P}^{D+1}, \quad D \geqslant 3,
$$

which turn out very simple and quite similar to special octics (see Theorem 5.11; the case $D=2$ is considered in [3]). The conjectural bound of 144 lines in double planes $X \rightarrow \mathbb{P}^{2}$ (see [3]) is left to a subsequent paper.

As a by-product of the classification of large Fano graphs, we obtain bounds on the maximal number $M_{\mathbb{R}}(D)$ of real lines in a real $K 3$-surface.

Theorem 1.4 (see Sect. 8.5) The number $M_{\mathbb{R}}(D)$ is as given by Table 1. If $D$ is not found in the table, then $M_{\mathbb{R}}(D) \leqslant 24$; this bound is sharp for $D \leqslant 16$.

\subsection{Asymptotic Bounds}

If $D$ is large, all Fano graphs become very simple, viz. disjoint unions of (affine) Dynkin diagrams, and we have

$$
\limsup _{D \rightarrow \infty} M_{\mathbb{C}}(D)=24, \quad \limsup _{D \rightarrow \infty} M_{\mathbb{R}}(D)=21 .
$$

More precisely, we have the following two asymptotic statements.

Theorem 1.5 (see Sect. 3.4) Let $X \subset \mathbb{P}^{D+1}$ be a smooth 2D-polarized K3-surface, and assume that $D \gg 0$. Then, either

- Fn $X$ is a disjoint union of Dynkin diagrams, and $|\mathrm{Fn} X| \leqslant 19$, or

- all lines in $X$ are fiber components of an elliptic pencil, and $|\mathrm{Fn} X| \leqslant 24$.

The precise sharp bound $M_{\mathbb{C}}(D), D \gg 0$, is as follows:

- if $D=1 \bmod 3$ or $D=2 \bmod 4$, then $M_{\mathbb{C}}(D)=24$;

- otherwise, if $D=0 \bmod 4$ or $D=2 \bmod 5$, then $M_{\mathbb{C}}(D)=22$;

- in all other cases, $M_{\mathbb{C}}(D)=21$.

Theorem 1.6 (see Sect. 3.5) If $D \gg 0$, the sharp bound $M_{\mathbb{R}}(D)$ is as follows:

- if $D=0 \bmod 3, D=1 \bmod 5$, or $D=0 \bmod 7$, then $M_{\mathbb{R}}(D)=21$;

- otherwise, if $D \notin\{17,113 \bmod 120\}$, then $M_{\mathbb{R}}(D)=20$;

- in all other cases, $M_{\mathbb{R}}(D)=19$.

The conclusion of Theorem 1.5 holds to full extent (all Fano graphs are elliptic or parabolic) for $D>620$ (see Remark 3.10). The expression for $M_{\mathbb{C}}(D)$ is valid for $D>45$ (see Corollary 4.8), and the expression for $M_{\mathbb{R}}(D)$ in Theorem 1.6 is valid for $D>257$ (cf. Corollary 4.9).

The structure of elliptic pencils carrying many lines is given by Corollary 3.8 and Table 2. 


\subsection{Discriminant Minimizing Surfaces}

Given a degree $h^{2}=2 D$, one can pose a question on the minimal discriminant

$$
\operatorname{md}(D):=\min \left\{\operatorname{det} T \mid \operatorname{rk} T=2 \text { and there is a smooth model } X(T) \subset \mathbb{P}^{D+1}\right\}
$$

of a singular $K 3$-surface $X(T)$ admitting a smooth model of degree $2 D$. In [3], this question was answered for $D=2,3,4$, and the conjecture on the maximal number of lines in sextic and octic models was based on the assumption that the surfaces minimizing the discriminant should also maximize the number of lines. Ironically, although the statement of the conjecture does hold, its motivating assumption fails for the very next value $D=5$.

The proof of the next theorem is omitted as it repeats, almost literally, the same computation as in [3].

Theorem 1.7 For each degree $h^{2}=2 D$ as in Table 1, there is a unique genus of discriminant minimizing transcendental lattices $T_{0}$. If $D \neq 5,10$, the discriminant minimizing surfaces have smooth models $X\left(T_{0}\right) \subset \mathbb{P}^{D+1}$ maximizing the number of lines (underlined in the table). For the two exceptional values, one has:

- $\operatorname{md}(5)=32$ and $X([2,0,16]) \subset \mathbb{P}^{6}$ has 28 lines (see $\Phi_{28}$ in [4]);

- $\operatorname{md}(10)=15$ and $X([4,1,4]) \subset \mathbb{P}^{11}$ has 24 (see $\Delta_{24}$ in [4]) or 20 lines.

Unless $D=3$ or 9 , the discriminant minimizing transcendental lattices $T_{0}$ are shown in Table 1 . If $D=3$, one has $T_{0}=[2,1,20]$ or $[6,1,8]$ and $X\left(T_{0}\right) \in \mathcal{M}_{3}\left(\Psi_{42}\right)$ (see [3]); if $D=9$, then $T_{0}=[2,1,8]$ and $X\left(T_{0}\right) \in \mathcal{M}_{9}\left(\Phi_{25}\right)$.

\subsection{Contents of the Paper}

In Sect. 2, after introducing the necessary definitions and results concerning lattices, polarized lattices and their relation to $K 3$-surfaces, and graphs, we state the arithmetical reduction of the line counting problem that is used throughout the paper. An important statement is Proposition 2.8: it rules out most hyperbolic graph for most degrees. As an application, in Sect. 2.5 we prove several sharp bounds on the valency of a line in a smooth polarized $K 3$-surface.

In Sect. 3, we discuss parabolic graphs (essentially, affine Dynkin diagrams) and their relation to elliptic pencils on a $K 3$-surface. As a counterpart of Proposition 2.8, we prove Proposition 3.7 stating the periodicity of the set of geometric degrees for each parabolic graph. In Sect. 3.4, we prove Theorems 1.5 and 1.6.

Having the conceptual part settled, we start the classification of hyperbolic Fano graphs. We divide them into groups, according to the minimal fiber, and study caseby-case: locally elliptic (see Sect. 4), astral (see Sect. 5), pentagonal (see Sect. 6), quadrangular (see Sect. 7), and triangular (see Sect. 8). For each group, we obtain a finer classification of large graphs in small degrees, which is stated as a separate theorem. Combining these partial statements, we prove Theorems 1.1 and 1.4 in Sect. 8.4.

In Sect. 5, we discuss also special octics and hyperelliptic models of $K 3$-surfaces. 
In Appendix A, we outline a few technical details concerning the algorithms used in the proofs when enumerating large Fano graphs.

\section{Preliminaries}

We start with an arithmetical reduction of the line counting problem (which, in fact, is well known, see Theorems 2.2 and 2.7) and describe the technical tools used to detect geometric configurations of lines. Then, Fano graphs are subdivided into elliptic, parabolic, and hyperbolic. The first two classes are not very interesting, as they may contain at most 19 or 24 lines, respectively. (Parabolic graphs are treated in more detail in Sect. 3.) Thus, the rest of the paper deals mainly with hyperbolic Fano graphs. The very first results, obtained in this section, are a degree bound (see Proposition 2.8) and valency bounds (see Propositions 2.10 and 2.11).

\subsection{Lattices}

A lattice is a finitely generated free abelian group $L$ equipped with a symmetric bilinear form $b: L \otimes L \rightarrow \mathbb{Z}$ (which is usually understood and omitted from the notation). We abbreviate $x \cdot y:=b(x, y)$ and $x^{2}:=b(x, x)$. In this paper, all lattices are even, i.e., $x^{2}=0 \bmod 2$ for all $x \in L$.

The determinant det $L$ is the determinant of the Gram matrix of $b$ in any integral basis, and the kernel $\operatorname{ker} L$ (as opposed to the $\operatorname{kernel} \operatorname{Ker} \varphi$ of a homomorphism $\varphi$ ) is the subgroup

$$
\operatorname{ker} L:=L^{\perp}=\{x \in L \mid x \cdot y=0 \text { for all } y \in L\} \text {. }
$$

An integral lattice $L$ is unimodular if $\operatorname{det} L= \pm 1$; it is nondegenerate if $\operatorname{det} L \neq 0$ or, equivalently, $\operatorname{ker} L=0$.

The inertia indices $\sigma_{ \pm, 0}(L)$ are the classical inertia indices of the quadratic form $L \otimes \mathbb{R}$. Clearly, $\sigma_{0}(L)=$ rk ker $L$. The inertia index $\sigma_{+}(L)\left(\right.$ the sum $\left.\sigma_{+}(L)+\sigma_{0}(L)\right)$ is the dimension of maximal positive definite (respectively, semidefinite) subspaces in $L \otimes \mathbb{R}$; a similar statement holds for $\sigma_{-}(L)$ and negative subspaces. Hence, these quantities are monotonous:

$$
\text { if } S \subset L \text {, then } \sigma_{ \pm}(S) \leqslant \sigma_{ \pm}(L) \text { and } \sigma_{ \pm}(S)+\sigma_{0}(S) \leqslant \sigma_{ \pm}(L)+\sigma_{0}(L) \text {. }
$$

A lattice $L$ is called hyperbolic if $\sigma_{+}(L)=1$.

An extension of a lattice $L$ is any overlattice $S \supset L$. If $L$ is nondegenerate, there is a canonical inclusion of $L$ to the dual group

$$
L^{\vee}=\{x \in L \otimes \mathbb{Q} \mid x \cdot y \in \mathbb{Z} \text { for all } y \in L\},
$$

which inherits from $L$ a $\mathbb{Q}$-valued quadratic form. Any finite index extension of $L$ is a subgroup of $L^{\vee}$. In general, extensions of $L$ can be described in terms of the discriminant group $\operatorname{discr} L:=L^{\vee} / L$ : this is a finite abelian group equipped with the 
nondegenerate $(\mathbb{Q} / 2 \mathbb{Z})$-valued quadratic form $(x \bmod L) \mapsto x^{2} \bmod 2 \mathbb{Z}$. Since we omit the details of the computation, we merely refer to the original paper [9].

\subsection{Polarized Lattices}

Given an even integer $D \geqslant 2$, a $2 D$-polarized lattice is a nondegenerate hyperbolic lattice $S$ equipped with a distinguished vector $h \in S$ such that $h^{2}=2 D$. For such a lattice $(S, h)$ we can define its set of lines

$$
\operatorname{Fn}(S, h):=\left\{v \in S \mid v^{2}=-2, v \cdot h=1\right\}
$$

This set is finite. Both $D$ and $h$ are often omitted from the notation. A polarized lattice $(S, h)$ is called a configuration if it is generated over $\mathbb{Q}$ by $h$ and $\operatorname{Fn}(S, h)$.

Denote by $\mathbf{L}$ a fixed representative of the isomorphism class of the intersection lattice $H_{2}(X ; \mathbb{Z})$ of a $K 3$-surface $X$ : this is the only (up to isomorphism) unimodular even lattice of rank 22 and signature -16 ; in other words, $\mathbf{L} \cong \mathbf{U}^{3} \oplus 2 \mathbf{E}_{8}^{2}(-1)$.

Definition 2.1 Depending on the geometric problem, we define "bad" vectors in a polarized lattice $(S, h)$ as vectors $e \in S$ with one of the following properties:

(1) $e^{2}=-2$ and $e \cdot h=0$ (exceptional divisor),

(2) $e^{2}=0$ and $e \cdot h=2$ (quadric pencil), or

(3) $e^{2}=0$ and $e \cdot h=3$ (cubic pencil).

Usually, only vectors as in (1) or (2) are excluded, whereas vectors as in (3) are to be excluded if $D=4$ and we consider triquadrics rather than all octic surfaces.

Respectively, a polarized lattice $(S, h)$ is called

- admissible, if it contains no "bad" vectors,

- geometric, if it is admissible and has a primitive embedding to $\mathbf{L}$, and

- subgeometric, if it admits a geometric finite index extension.

Given a smooth $2 D$-polarized $K 3$-surface $X \subset \mathbb{P}^{D+1}$, we denote by Fn $X$ the set of lines contained in $X$ (the Fano graph) and define the Fano configuration $\mathcal{F}_{h}(X)$ as the primitive sublattice $S \subset H_{2}(X ; \mathbb{Z})$ generated over $\mathbb{Q}$ by the polarization $h$ and the classes $[l]$ of all lines $l \in \mathrm{Fn} X$. The next statement is standard and well known; it follows from the global Torelli theorem for $K 3$-surfaces [11], surjectivity of the period map [8], and Saint-Donat's results on projective models of $K 3$-surfaces [14] (cf. also [5, Thm. 3.11] or [3, Thm. 7.3]).

Theorem 2.2 A 2D-configuration $(S, h)$ is isomorphic to the Fano configuration $\mathcal{F}_{h}(X)$ of a smooth 2D-polarized K3-surface $X$ if and only if $(S, h)$ is geometric; in this case, an isomorphism $(S, h) \rightarrow \mathcal{F}_{h}(X)$ induces a bijection $\operatorname{Fn}(S, h) \rightarrow \operatorname{Fn} X$.

Given a primitive embedding $S \hookrightarrow \mathbf{L}$, all $2 D$-polarized surfaces $X$ such that

$$
\left(H_{2}(X ; \mathbb{Z}), \mathcal{F}_{h}(X), h\right) \cong(\mathbf{L}, S, h)
$$

constitute one or two (complex conjugate) equilinear deformation families; modulo the projective group $P G L(D+2, \mathbb{C})$, their dimension equals $20-\mathrm{rk} S$. 
Remark 2.3 The number of families in Theorem 2.2 equals one or two depending on whether $\mathbf{L}$ does or, respectively, does not admit an automorphism preserving $h$ and $S$ (as a set) and reversing the orientation of maximal positive definite subspaces in $\mathbf{L} \otimes \mathbb{R}$. (Such an automorphism would be induced by the complex conjugation.) A configuration $S$ is called rigid if rk $S=20$ : such configurations are realized by finitely many projective equivalence classes of surfaces.

Remark 2.4 According to [14], if $D=4$, one should also require that $h \in S$ is a primitive vector. However, this condition holds automatically if Fn $S \neq \varnothing$. Besides, in this case one can also distinguish between all octic surfaces and triquadrics; as explained above, the difference is in the definition of the admissibility: cubic pencils as in Definition 2.1(3) are either allowed (special octics) or excluded (triquadrics).

We use Theorem 2.2 in conjunction with the following two algorithms.

Algorithm 2.5 There is an effective algorithm (using the enumeration of vectors of given length in a definite lattice, implemented as ShortestVectors in GAP [7]) detecting whether a lattice $(S, h)$ is admissible and computing the $\operatorname{set} \operatorname{Fn}(S, h)$.

Algorithm 2.6 A nondegenerate lattice admits but finitely many finite index extensions, which can be effectively enumerated (see [9, Prop. 1.4.1]). Then, one can use Algorithm 2.5 to select the admissible extensions (note that this is not automatic) and, for each such extension, use [9, Corr. 1.12.3] to detect whether it admits a primitive embedding to $\mathbf{L}$. (A necessary, but not sufficient condition is that rk $S \leqslant 20$.) This computation, using Nikulin's techniques of discriminant forms, can also be implemented in GAP [7].

\subsection{Real Configurations}

For a real (i.e., invariant under the standard complex conjugation involution) smooth $K 3$-surface $X \subset \mathbb{P}^{D+1}$, denote by $\mathrm{Fn}_{\mathbb{R}} X \subset$ Fn $X$ the set of real lines contained in $X$. As explained in [5], $X$ can be deformed to a real surface $X^{\prime}$ such that $\mathrm{Fn} X^{\prime}=\mathrm{Fn}_{\mathbb{R}} X^{\prime} \cong$ $\mathrm{Fn}_{\mathbb{R}} X$, i.e., all lines in $X^{\prime}$ are real. Hence, when computing the bound $M_{\mathbb{R}}(D)$, we can assume all lines real.

Theorem 2.7 (cf. [5, Lem. 3.10]) Consider a geometric configuration $(S, h)$ and a primitive embedding $S \hookrightarrow \mathbf{L}$. Then, family (2.2) contains a real surface with all lines real if and only if the generic transcendental lattice $T:=S^{\perp}$ has a sublattice isomorphic to [2] or $\mathbf{U}(2)$.

\subsection{Configurations as Graphs}

One can easily show that, if a polarized lattice $S$ is admissible, for any two lines $u, v \in \mathrm{Fn} S$ one has $u \cdot v=0$ or 1 ; respectively, we say that $u$ and $v$ are disjoint or intersect. Therefore, it is convenient to regard Fn $S$ as a graph: the vertices are the lines $v \in \mathrm{Fn} S$, and two vertices are connected by an edge if and only if the lines intersect. 
We adopt the graph theoretic terminology; for example, we define the valency val $v$ of a line $v \in$ Fn $S$ as the number of lines $u \in$ Fn $S$ such that $u \cdot v=1$.

The term subgraph always means an induced subgraph. To simplify statements, introduce the relative valency

$$
\operatorname{val}_{\Sigma} l:=\#\{a \in \Sigma \mid a \cdot l=1\}
$$

with respect to a subgraph $\Sigma \subset \Gamma$ and, given two subgraphs $\Sigma_{1}, \Sigma_{2} \subset \Gamma$, define

$$
\Sigma_{1} * \Sigma_{2}:=\max \left\{\operatorname{val}_{\Sigma_{i}} l_{j} \mid l_{j} \in \Sigma_{j},(i, j)=(1,2) \text { or }(2,1)\right\}
$$

For example, $\Sigma * \Sigma=0$ if and only if the subgraph $\Sigma \subset \Gamma$ is discrete.

Conversely, to an abstract graph $\Gamma$ (loop free and without multiple edges) we can associate three lattices

$$
\mathbb{Z} \Gamma, \quad \mathcal{F}(\Gamma):=\mathbb{Z} \Gamma / \text { ker, } \quad \mathcal{F}_{h}(\Gamma):=(\mathbb{Z} \Gamma+\mathbb{Z} h) / \text { ker. }
$$

Here, $\mathbb{Z} \Gamma$ is freely generated by the vertices $v \in \Gamma$, so that $v^{2}=-2$ and $u \cdot v=1$ or 0 if $u$ and $v$ are, respectively, adjacent or not. For the last lattice $\mathcal{F}_{h}(\Gamma)$, the sum is direct, but not orthogonal; the even integer $2 D:=h^{2} \geqslant 4$ is assumed fixed in advance, and we have $v \cdot h=1$ for each $v \in \Gamma$.

A graph $\Gamma$ is called

- elliptic, if $\sigma_{+}(\mathbb{Z} \Gamma)=\sigma_{0}(\mathbb{Z} \Gamma)=0$,

- parabolic, if $\sigma_{+}(\mathbb{Z} \Gamma)=0$ and $\sigma_{0}(\mathbb{Z} \Gamma)>0$, and

- hyperbolic, if $\sigma_{+}(\mathbb{Z} \Gamma)=1$.

(Since we are interested in hyperbolic lattices only, we do not consider graphs with $\sigma_{+}(\mathbb{Z} \Gamma)>1$.) When the degree $h^{2}=2 D$ is fixed, we extend to graphs the terms admissible, geometric, and subgeometric introduced in Definition 2.1, referring to the corresponding properties of the lattice $\mathcal{F}_{h}(\Gamma)$. By Theorem 2.2, subgeometric graphs are those that can appear as induced subgraphs in the Fano graph Fn $X$ of a smooth $2 D$-polarized $K 3$-surface $X$. Conversely, a degree $D$ is geometric for $\Gamma$ if $\Gamma \cong \operatorname{Fn} X$ for some smooth $2 D$-polarized $K 3$-surface $X$; the set of geometric degrees is denoted by $\operatorname{gd} \Gamma$. Similarly, for a subfield $K \subset \mathbb{C}$, we introduce the set $\operatorname{gd}_{K} \Gamma$ of the values of $D$ for which $\Gamma \cong \mathrm{Fn}_{K} X$ for some smooth $2 D$-polarized $K 3$-surface $X \hookrightarrow \mathbb{P}^{D+1}$ defined over $K$.

The correspondence between graphs and lattices is not exactly one-to-one: in general, $S \supset \mathcal{F}_{h}(\operatorname{Fn} S)$ is a finite index extension and $\Gamma \subset \operatorname{Fn} \mathcal{F}_{h}(\Gamma)$ is an induced subgraph (assuming that $\mathcal{F}_{h}(\Gamma)$ is hyperbolic).

If $\Gamma$ is elliptic, the lattice $\mathcal{F}_{h}(\Gamma)$ is obviously hyperbolic. Parabolic graphs are treated separately in Sect. 3.1. If $\Gamma$ is hyperbolic, we can define the intrinsic polarization as a vector $h_{\Gamma} \in \mathbb{Z} \Gamma \otimes \mathbb{Q}$ with the property that $v \cdot h_{\Gamma}=1$ for each vertex $v \in \Gamma$. If such a vector exists, it is unique modulo ker $\mathbb{Z} \Gamma$; in particular, $h_{\Gamma}^{2} \in \mathbb{Q}$ is well defined. (Note that $h_{\Gamma}^{2}$ may be negative.)

Proposition 2.8 Given a hyperbolic graph $\Gamma$, the $2 D$-polarized lattice $\mathcal{F}_{h}(\Gamma)$ is hyperbolic if and only if $h_{\Gamma}$ exists and $2 D \leqslant h_{\Gamma}^{2}$. 
Proof Assume that $\mathcal{F}_{h}(\Gamma)$ is hyperbolic. Then, by (2.1), the image of the projection $\varphi: \mathbb{Z} \Gamma \rightarrow \mathcal{F}_{h}(\Gamma)$ is nondegenerate and we have the orthogonal projection

$$
\operatorname{pr}: \mathcal{F}_{h}(\Gamma) \rightarrow \varphi(\mathbb{Z} \Gamma) \otimes \mathbb{Q}
$$

Thus, $h_{\Gamma}$ exists (as any pull-back of $\operatorname{pr} h$ ) and, since $\varphi(\mathbb{Z} \Gamma)$ itself is hyperbolic, its orthogonal complement must be negative definite, i.e., $h^{2} \leqslant h_{\Gamma}^{2}$ (and, if the equality holds, we must have $h \in \varphi(\mathbb{Z} \Gamma) \otimes \mathbb{Q})$.

The converse statement is straightforward: if $h_{\Gamma}$ exists, we have an orthogonal direct sum decomposition $\mathcal{F}_{h}(\Gamma) \otimes \mathbb{Q}=(\mathcal{F}(\Gamma) \otimes \mathbb{Q}) \oplus \mathbb{Q} v$, where $v:=h-\varphi\left(h_{\Gamma}\right)$, in which either $v=0$ (if $2 D=h_{\Gamma}^{2}$ ) or $v^{2}<0$.

Corollary 2.9 (of the proof) Let $\Gamma$ be a hyperbolic graph and $\operatorname{rk} \mathcal{F}(\Gamma)=20$. Then, the $2 D$-polarized lattice $\mathcal{F}_{D}(\Gamma)$ can be geometric only if $2 D=h_{\Gamma}^{2}$.

Thus, each hyperbolic graph $\Gamma$ can be contained in the Fano graph of a smooth $2 D$-polarized $K 3$-surface $X$ only for finitely many values of $D$. Some of the values allowed by Proposition 2.8 can further be eliminated by requiring that $\Gamma$ should be subgeometric and using Algorithms 2.5 and 2.6. Below, for each graph $\Gamma$ used, we merely describe the set $\mathrm{gd} \Gamma$, referring to Proposition 2.8 and omitting further details of the computation.

\subsection{Valency Bounds}

Applying Proposition 2.8 to a star-shaped graph with a "central" vertex $v$ of valency val $v \geqslant 5$, we obtain the following statement.

Proposition 2.10 Let $X \subset \mathbb{P}^{D+1}$ be a smooth $2 D$-polarized K3-surface, fix a line $v \in \mathrm{Fn} X$, and assume that all lines intersecting $v$ are pairwise disjoint. Then, we have the following sharp bounds on val $v$ :

$$
\begin{array}{ccccccccc}
D & = & 2 & 3 & 4 & 5 & 6 & 7 . .11 & \text { else } \\
\text { val } v \leqslant & 12 & 9 & 8 & 7 & 6 & 5 & 4
\end{array}
$$

If $D=4$ and $X$ is a triquadric, then val $v \leqslant 7$ (sharp bound).

In Proposition 2.10, if $D=3$ and $v$ intersects ten disjoint lines $a_{1}, \ldots a_{10}$, then there is an eleventh line $b$ intersecting $v$ and one of $a_{i}$, cf. Proposition 2.11.

Without the assumption that the star star $v \subset$ Fn $X$ is discrete, the bound for quartics $(D=2)$ is val $v \leqslant 20$, and star $v$ may be rather complicated (see [5,12]). The star simplifies dramatically if $D \geqslant 3$ : the following statement is also proved by applying Proposition 2.8 to a few simple test configurations.

Proposition 2.11 Let $X \subset \mathbb{P}^{D+1}$ be a smooth $2 D$-polarized $K 3$-surface, $D \geqslant 3$, and let $v, a_{1}, a_{2} \in \mathrm{Fn} X$ be three lines such that $v \cdot a_{1}=v \cdot a_{2}=a_{1} \cdot a_{2}=1$. Then, all other lines intersecting $v$ are disjoint from $a_{1}, a_{2}$, and each other and we have the following sharp bounds on val $v$ : 


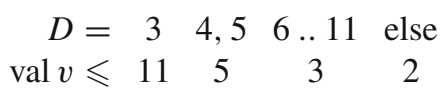

Furthermore, if $D=3$, then val $v \neq 10$.

$\triangleleft$

\section{Elliptic Pencils}

In this section, we establish a relation between parabolic Fano graphs Fn $X$ and elliptic pencils on the $K 3$-surface $X$. In particular, we establish a certain periodicity for such graphs (see Proposition 3.7) and prove Theorems 1.5 and 1.6.

\subsection{Parabolic Graphs}

It is well known that any parabolic or elliptic graph $\Gamma$ is a disjoint union of simply laced Dynkin diagrams and affine Dynkin diagrams, with at least one component affine if $\Gamma$ is parabolic. We describe the combinatorial type of $\Gamma$ as a formal sum of the corresponding $\mathbf{A}-\mathbf{D}-\mathbf{E}$ types of its components.

If $\Sigma$ is a connected parabolic graph (affine Dynkin diagram), the kernel ker $\mathbb{Z} \Sigma$ is generated by a single vector $k_{\Sigma}=\sum n_{v} v, v \in \Sigma$; this generator can be chosen so that all $n_{v}>0$ and, under this assumption, it is unique. We call the coefficient sum $\operatorname{deg} \Sigma:=\sum_{v} n_{v}$ the degree of $\Sigma$. We have

$$
\begin{gathered}
\operatorname{deg} \widetilde{\mathbf{A}}_{p}=p+1, \quad p \geqslant 1, \quad \operatorname{deg} \widetilde{\mathbf{D}}_{q}=2 q-2, q \geqslant 4, \\
\operatorname{deg} \widetilde{\mathbf{E}}_{6}=12, \quad \operatorname{deg} \widetilde{\mathbf{E}}_{7}=18, \quad \operatorname{deg} \widetilde{\mathbf{E}}_{8}=30
\end{gathered}
$$

For a connected elliptic graph (Dynkin diagram) $\Sigma$, we can define the degree set ds $\Sigma$ as the set $\left\{\sum_{v} m_{v}\right\}$ of the coefficient sums of all positive roots $\sum_{v} m_{v} v \in \mathbb{Z} \Sigma$. A simple computation shows that

$$
\begin{gathered}
\mathrm{ds} \mathbf{A}_{p}=\{1 . . p\}, \quad p \geqslant 1, \quad \mathrm{ds} \mathbf{D}_{q}=\{1 . .2 q-3\}, q \geqslant 4, \\
\mathrm{ds} \mathbf{E}_{6}=\{1 . .11\}, \quad \mathrm{ds} \mathbf{E}_{7}=\{1 . .13,17\}, \quad \operatorname{ds} \mathbf{E}_{8}=\{1 . .16,23\} .
\end{gathered}
$$

The Milnor number of a parabolic or elliptic graph $\Gamma$ is $\mu(\Gamma):=\operatorname{rk} \mathcal{F}(\Gamma)$. We have

$$
|\Gamma|=\mu(\Gamma)+\#(\text { parabolic components of } \Gamma) \text {. }
$$

Lemma 3.1 Let $\Sigma$ be a parabolic component of an admissible parabolic graph $\Gamma$. Then, the image of $\operatorname{ker} \mathbb{Z} \Gamma$ under the projection $\varphi: \mathbb{Z} \Gamma \rightarrow \mathcal{F}_{h}(\Gamma)$ is generated by the image $\varphi\left(k_{\Sigma}\right) \neq 0$, which is primitive in any admissible extension $S \supset \mathcal{F}_{h}(\Gamma)$.

Proof Since $\mathcal{F}_{h}(\Gamma)$ is assumed hyperbolic, by $(2.1)$ one has $\operatorname{rk} \varphi(\operatorname{ker} \mathbb{Z} \Gamma) \leqslant 1$. On the other hand, $\varphi\left(k_{\Sigma}\right) \cdot h=\operatorname{deg} \Sigma \neq 0$; hence, $\varphi\left(k_{\Sigma}\right) \neq 0$ and this vector generates $\varphi(\operatorname{ker} \mathbb{Z} \Gamma)$ over $\mathbb{Q}$. To show that $\varphi\left(k_{\Sigma}\right) \neq 0$ is primitive, consider a maximal elliptic subgraph $\Sigma_{0} \subset \Sigma$ : it is a Dynkin diagram of the same name. If $a:=\frac{1}{m} k_{\Sigma} \in S$ for some $m \geqslant 2$, then, by (3.1) and (3.2), $a \cdot h \in \mathrm{ds} \Sigma_{0}$; hence, there is a root $e \in \mathbb{Z} \Sigma_{0}$ with $e \cdot h=a \cdot h$, and $e-a$ is an exceptional divisor as in Definition 2.1(1). 
Lemma 3.1 implies that $\varphi$ maps elements $k_{\Sigma} \in \mathbb{Z} \Sigma$ corresponding to all parabolic components $\Sigma$ of $\Gamma$ to the same element of $\mathcal{F}_{h}(\Gamma)$, viz. positive primitive generator of the kernel $\operatorname{ker} \varphi(\mathbb{Z} \Gamma)$.

Corollary 3.2 In any admissible graph $\Gamma$, one has $v \cdot k_{\Sigma^{\prime}}=v \cdot k_{\Sigma^{\prime \prime}}$ for any two disjoint connected parabolic subgraphs $\Sigma^{\prime}, \Sigma^{\prime \prime} \subset \Gamma$ and any vector $v \in \mathcal{F}_{h}(\Gamma)$.

Corollary 3.3 All parabolic components of an admissible parabolic graph $\Gamma$ have the same degree; this common degree deg $\Gamma$ is called the degree of $\Gamma$.

An admissible graph $\Gamma$ is called saturated if $\Gamma=\operatorname{Fn} \mathcal{F}_{h}(\Gamma)$. (A priori, this notion depends on the choice of a degree $h^{2}=2 D$.) The following statement is proved similar to the primitivity of $\varphi\left(k_{\Sigma}\right)$ in Lemma 3.1.

Corollary 3.4 If $\Gamma$ is an admissible parabolic graph and $\Sigma$ is an elliptic component of $\Gamma$, then $\operatorname{deg} \Gamma \notin \mathrm{ds} \Sigma$. If $\Gamma$ is saturated, then also $(\operatorname{deg} \Gamma-1) \notin \mathrm{ds} \Sigma$.

\subsection{Parabolic Graphs as Elliptic Pencils}

Let $X$ be a smooth $2 D$-polarized $K 3$-surface, and consider a connected parabolic subgraph $\Sigma \subset$ Fn $X$. (Recall that $\Sigma$ is an affine Dynkin diagram.) The class $k_{\Sigma}$, regarded as a divisor, is nef and, since also $k_{\Sigma}^{2}=0$ and $k_{\Sigma}$ is primitive in $H_{2}(X ; \mathbb{Z})$ (see Lemma 3.1), this class is a fiber of a certain elliptic pencil $\pi: X \rightarrow \mathbb{P}^{1}$. We denote by $\mathcal{P}(\Sigma) \subset$ Fn $X$ the subgraph spanned by all linear components of the reducible fibers of $\pi$. Clearly,

$$
\mathcal{P}(\Sigma)=\left\{v \in \operatorname{Fn} X \mid v \cdot k_{\Sigma}=0\right\}
$$

alternatively, $\mathcal{P}(\Sigma)$ consists of $\Sigma$ and all vertices $v \in$ Fn $X$ that are not adjacent to any of the vertices of $\Sigma$. In this form, the notion of pencil can be extended to any geometric configuration $S$ and affine Dynkin diagram $\Sigma \subset$ Fn $S$; we use the same notation $\mathcal{P}(\Sigma)$, or $\mathcal{P}_{S}(\Sigma)$ if $S$ is to be specified.

Note that $\mathcal{P}(\Sigma)$ is a parabolic graph and $\mathcal{P}(\Sigma)=\mathcal{P}\left(\Sigma^{\prime}\right)$ for any other parabolic component $\Sigma^{\prime} \subset \mathcal{P}(\Sigma)$. In this language, $\operatorname{deg} \mathcal{P}(\Sigma)$ is the common projective degree of the fibers of $\pi$.

Thus, any maximal geometric parabolic graph $\Gamma$ is the set of linear components of the fibers of an elliptic pencil. By Lemma 3.1 and (2.1), we have

$$
\mu(\Gamma)=|\Gamma|-\#(\text { parabolic components of } \Gamma) \leqslant 18 \text {. }
$$

Besides, the usual bound on the topological Euler characteristic yields

$$
|\Gamma| \leqslant \chi(X)=24
$$

with the inequality strict whenever $\Gamma$ has at least one elliptic component. Under the additional assumption that $\operatorname{deg} \Gamma \leqslant 7$, this can be refined to

$$
\mu(\Gamma)+b_{0}(\Gamma)=|\Gamma|+\#(\text { elliptic components of } \Gamma) \leqslant 24
$$


by taking into account the fiber components of higher projective degree. (Indeed, considering singular elliptic fibers one by one, one can easily see that, under the assumption $\operatorname{deg} \Gamma \leqslant 7$, the number of elliptic components of $\Gamma$ does not exceed the number of fiber components of $\pi$ that are not lines and, thus, are not present in $\Gamma$. On the other hand, the total number of fiber components is at most 24.) The two latter bounds are less obvious arithmetically; their proof would require considering graphs satisfying (3.3) one by one and using Algorithm 2.6 (cf. [5]).

Remark 3.5 Without the assumption $\operatorname{deg} \Gamma \leqslant 7$, the a priori possible pencils $\Gamma$ of a given degree $d$ can be enumerated as follows. Consider a collection $\Sigma_{1}, \ldots, \Sigma_{n}$ of affine Dynkin diagrams such that $\sum_{i}\left|\Sigma_{i}\right| \leqslant 24$ and assign an integral weight (projective degree) $w_{v} \geqslant 1$ to each vertex $v$ of the union so that $\sum_{v \in \Sigma} n_{v} w_{v}=d$ for each component $\Sigma=\Sigma_{i}$, where $\sum_{v \in \Sigma} n_{v} v=k_{\Sigma}$. Then, take for $\Gamma$ the induced subgraph of the union $\bigcup_{i} \Sigma_{i}$ spanned by all vertices of weight 1 . (Note that we have $\operatorname{deg} \Sigma_{i} \leqslant d$ for each $i$ and, for $\Gamma$ to be parabolic, the equality must hold for at least one value of $i$.) This description does not guarantee that $\Gamma$ is geometric, but it rules out many graphs that are not.

To emphasize the relation between parabolic graphs and elliptic pencils, we adopt the following terminology. A maximal parabolic subgraph $\Gamma \subset$ Fn $X$ is called a pencil, and its components are called fibers; a pencil is uniquely determined by any of its parabolic fibers. (Strictly speaking, only parabolic components of $\Gamma$ are whole fibers of $\pi$, whereas elliptic ones are parts of fibers containing irreducible curves of higher projective degree; it may even happen that several elliptic components of $\Gamma$ are contained in the same fiber of $\pi$.) All other lines in $X$ are called sections of $\Gamma$ : they are indeed (multi-)sections of $\pi$, and we disregard all multisections of higher projective degree. We denote by $\sec \Gamma:=\operatorname{Fn} X \backslash \Gamma$ the set of all sections, and

$$
\sec _{n} \Gamma:=\left\{v \in \operatorname{Fn} X \mid v \cdot k_{\Sigma}=n \text { for some/any parabolic fiber } \Sigma \text { of } \Gamma\right\}
$$

stands for the set of $n$-sections, $n \geqslant 1$ (see Corollary 3.2). For $l \in \Gamma$, we also use

$$
\sec l:=\{v \in \operatorname{Fn} X \backslash \Gamma \mid v \cdot l=1\} \quad \text { and } \sec _{n} l:=\sec l \cap \sec _{n} \Gamma .
$$

Sometimes, 1- and 2-sections are called simple sections and bisections, respectively. Any section of $\Gamma$ intersects each parabolic fiber, but it may miss elliptic ones.

As an immediate consequence of these definitions, we have

$$
|\operatorname{Fn} X|=|\Gamma|+|\sec \Gamma|
$$

for each pencil $\Gamma \subset$ Fn $X$.

Convention 3.6 A section $s \in \sec \Gamma$ can be described by its coordinates

$$
\bar{s}:=\{l \in \Gamma \mid s \cdot l=1\} \subset \Gamma .
$$

If $s$ is an $n$-section, then $\left|\bar{s} \cap \Sigma^{\prime}\right|=n$ and $\left|\bar{s} \cap \Sigma^{\prime \prime}\right| \leqslant n$ for each parabolic fiber $\Sigma^{\prime} \subset \Gamma$ and elliptic fiber $\Sigma^{\prime \prime} \subset \Gamma$, respectively. Often, the map $s \mapsto \bar{s}$ is injective, but 
we do not assert this in general. If a configuration $S$ is spanned by $h, \Gamma$, and a number of pairwise disjoint sections $s_{1}, \ldots, s_{n} \in \sec \Gamma$, the Gram matrix of $S$ is determined by the coordinates $\bar{s}_{1}, \ldots, \bar{s}_{n}$. That is why, in the rest of the paper, we pay special attention to finding as many a priori disjoint sections as possible.

\subsection{The Periodicity}

Denote by $\pi_{D}(\Gamma)$ the set of connected components of the equilinear stratum Fn $X \cong \Gamma$ of smooth $2 D$-polarized $K 3$-surfaces. The following statement is in sharp contrast with Proposition 2.8.

Proposition 3.7 Let $\Gamma$ be a parabolic graph, $d:=\operatorname{deg} \Gamma$, and let $D \geqslant d^{2}+d$. Then, for any $D^{\prime}=D \bmod d$, there is a canonical inclusion $\pi_{D^{\prime}}(\Gamma) \hookrightarrow \pi_{D}(\Gamma)$. This inclusion is a bijection if also $D^{\prime} \geqslant d^{2}+d$.

Proof Consider the sublattice $H:=\mathbb{Z} h+\mathbb{Z} k \subset \mathcal{F}_{h}(\Gamma)$, where $k$ is the common image of the vectors $k_{\Sigma} \in \mathbb{Z} \Sigma$ corresponding to all parabolic components $\Sigma$ of $\Gamma$. The change of variables $h \mapsto h \pm k$ shows that the abstract isomorphism type of the pair of lattices $H \subset \mathcal{F}_{h}(\Gamma)$ depends on $D$ mod $d$ only. Since any polarized automorphism of $\mathcal{F}_{h}(\Gamma)$ preserves $H$ pointwise, the set of isomorphism classes of embeddings $\mathcal{F}_{h}(\Gamma) \hookrightarrow \mathbf{L}$ also depends only on $D \bmod d$; hence, by Theorem 2.2 , we only need to investigate the dependence on $D$ of the admissibility of a finite index extension $S \supset \mathcal{F}_{h}(\Gamma)$ and the existence of extra lines.

Let $E:=H^{\perp}$; this is a negative definite lattice. We have $S \subset \mathcal{F}_{h}(\Gamma)^{\vee} \subset H^{\vee} \oplus E^{\vee}$ (cf. [9]), and any vector $e \in S$ decomposes as $e=e_{H}+e_{E}, e_{H} \in H^{\vee}, e_{E} \in E^{\vee}$. The sublattice $H$ is primitive: if $a:=\alpha h+\beta k \in \mathbf{L}$, we have $\alpha=a \cdot l \in \mathbb{Z}$ for any $l \in \Gamma$, and then $\beta \in \mathbb{Z}$ by Lemma 3.1. Hence, $e_{E}^{2}<0$ unless $e \in H$. Note also that $H^{\vee}$ is generated by $h^{*}=k / d$ and $k^{*}=(d h-2 D k) / d^{2}$.

Let $D \geqslant d^{2}+d$. If $e$ is an exceptional divisor as in Definition 2.1(1), then

$$
e_{H}=m k^{*}, m \in \mathbb{Z}, \quad e_{H}^{2}=-\frac{2 m^{2} D}{d^{2}}<-2
$$

unless $m=0$. It follows that $e \in E^{\vee}$ is independent of $D$. If $e$ is a quadric pencil as in Definition 2.1(2), then

$$
e_{H}=2 h^{*}+m k^{*}, m \in \mathbb{Z}, \quad e_{H}^{2}=-\frac{2 m(m D-2 d)}{d^{2}} \leqslant 0 .
$$

Hence, $m=0$ and $e=e_{H}=2 h^{*}$, but this vector cannot be in $S$ by the primitivity of $H$. Finally, if $e$ is a line, then

$$
e_{H}=h^{*}+m k^{*}, m \in \mathbb{Z}, \quad e_{H}^{2}=-\frac{2 m(m D-d)}{d^{2}}<-2
$$

unless $m=0$ or $m=1$ and $D=d^{2}+d$. In the latter case, $e_{H}^{2}=-2$ and, hence, $e=e_{H}=h^{*}+k^{*} \notin S$. In the former case, $e=h^{*}+e_{E}$, where $e_{E} \in E^{\vee}, e_{E}^{2}=-2$ 
Table 2 Large subgeometric parabolic graphs

\begin{tabular}{|c|c|c|c|}
\hline$|\Gamma|$ & $\Gamma$ & Polarizations $D$ & $T:=\mathcal{F}_{h}(\Gamma)^{\perp}$ \\
\hline \multirow[t]{2}{*}{24} & $8 \widetilde{\mathbf{A}}_{2}$ & $4^{+}$or $\{7 \bmod 3\}$ & $\mathbf{U}^{2}(3)$ \\
\hline & $6 \widetilde{\mathbf{A}}_{3}$ & $2^{+}$or $\{6 \bmod 4\}$ & {$[8,0,8]$} \\
\hline \multirow[t]{4}{*}{22} & $7 \widetilde{\mathbf{A}}_{2}+\mathbf{A}_{1}$ & $4^{+}$or $\{7 \bmod 3\}$ & $\mathbf{U}^{2}(3) \oplus[-6]$ \\
\hline & $5 \widetilde{\mathbf{A}}_{3}+\mathbf{A}_{2}$ & $2^{+}$or $\{6 \bmod 4\}$ & $\mathbf{A}_{2}(-4) \oplus[-16]$ \\
\hline & $5 \tilde{\mathbf{A}}_{3}+2 \mathbf{A}_{1}$ & $2^{+}$or $\{4 \bmod 2\}$ & {$[8,0,8] \oplus[-4]$} \\
\hline & $4 \widetilde{\mathbf{A}}_{4}+2 \mathbf{A}_{1}$ & $2^{+}$or $\{7 \bmod 5\}$ & {$[10,0,10]$} \\
\hline \multirow[t]{23}{*}{21} & $7 \tilde{\mathbf{A}}_{2}$ & $\{4 \bmod 3\}$ & $\mathbf{U}^{2}(3) \oplus \mathbf{A}_{2}(-3)$ \\
\hline & & $5^{+}$or $\{8 \bmod 3\}$ & $\mathbf{U}^{2}(3) \oplus \mathbf{A}_{2}(-1)$ \\
\hline & $6 \tilde{\mathbf{A}}_{2}+3 \mathbf{A}_{1}$ & $3^{+}$or $\{6 \bmod 3\}^{\ddagger}$ & $\mathbf{A}_{2} \oplus[-6]^{3}$ \\
\hline & & $\{4 \bmod 3\}$ & $\mathbf{A}_{2}(3) \oplus[-6]^{3}$ \\
\hline & $5 \tilde{\mathbf{A}}_{3}+\mathbf{A}_{1}$ & $\{2 \bmod 2\}$ & {$[12,4,12] \oplus[-4]^{2}$} \\
\hline & $4 \widetilde{\mathbf{A}}_{4}+\mathbf{A}_{1}$ & $\{2,5 \bmod 5\}$ & {$[50] \oplus \mathbf{U}(5)$} \\
\hline & & $6^{+}$or $\{11 \bmod 5\}^{+}$ & {$[2] \oplus \mathbf{U}(5)$} \\
\hline & $3 \widetilde{\mathbf{A}}_{5}+\mathbf{A}_{3}$ & $\{4 \bmod 6\}$ & {$[24,0,36]$} \\
\hline & & $2^{+}$or $\{8 \bmod 6\}$ & {$[4,0,24]$} \\
\hline & $3 \widetilde{\mathbf{A}}_{5}+3 \mathbf{A}_{1}$ & $\{4 \bmod 6\}$ & {$[24,12,24]$} \\
\hline & & $\{6 \bmod 6\}$ & {$[8,4,8]$} \\
\hline & $3 \tilde{\mathbf{A}}_{6}$ & $14^{+}$or $\{21 \bmod 7\}^{\ddagger}$ & {$[2,1,4]$} \\
\hline & & $\{2,4,8 \bmod 7\}$ & {$[14,7,28]$} \\
\hline & $2 \widetilde{\mathbf{D}}_{5}+\widetilde{\mathbf{A}}_{7}+\mathbf{A}_{1}$ & $\{2 \bmod 4\}$ & {$[32,16,40]$} \\
\hline & $3 \widetilde{\mathbf{D}}_{6}$ & $\{10 \bmod 10\}$ & {$[4,0,4],[20,0,20$} \\
\hline & & $\{2,8 \bmod 10\}$ & {$[4,0,100]$} \\
\hline & & $\{4,6 \bmod 10\}$ & {$[8,4,52]$} \\
\hline & $3 \widetilde{\mathbf{E}}_{6}$ & $\{4 \bmod 12\}$ & {$[6,0,72]$} \\
\hline & & $\{6 \bmod 12\}^{\ddagger}$ & {$[2,0,24]$} \\
\hline & & $\{7 \bmod 12\}$ & {$[6,0,18],[6,3,6]$} \\
\hline & & $\{10 \bmod 12\}$ & {$[18,0,24]$} \\
\hline & & $\{12 \bmod 12\}$ & {$[6,0,8]$} \\
\hline & & $\{15 \bmod 12\}^{\ddagger}$ & {$[2,0,6],[2,1,2]$} \\
\hline
\end{tabular}

does not depend on $D$. Thus, oll "undesirable" vectors are independent of the choice of $D \geqslant d^{2}+d$ within the same class $D \bmod d$. If $D<d^{2}+d$, these vectors are still present, but new ones can appear, ruling out some of the configurations.

Corollary 3.8 Table 2 lists all subgeometric pencils $\Gamma$ such that $|\Gamma| \geqslant 21$.

In the table, for each pencil $\Gamma$, we list the values of $D$ (marking with a ${ }^{+}$those for which any geometric finite index extension of $\mathcal{F}_{h}(\Gamma)$ contains sections of $\Gamma$ ) and generic transcendental lattices $T:=\mathcal{F}_{h}(\Gamma)^{\perp}$, which depend on $D \bmod \operatorname{deg} \Gamma$ only. 
Proof of Corollary 3.8 If $|\Gamma|=24$, then, by (3.3), $\Gamma$ has at least six parabolic fibers and, hence, the minimal Milnor number $\mu_{0}$ of such a fiber is subject to the inequality $6 \mu_{0} \leqslant 18$. Taking (3.5) into account, we arrive at the two combinatorial types listed in the table. Similarly, one can show that $|\Gamma| \neq 23$ and classify all pencils with $|\Gamma|=22$ or 21. (The combinatorial types that are never geometric are not shown in the table.) Finally, the set $\mathrm{gd} \Gamma$ for each combinatorial type $\Gamma$ is determined using Proposition 3.7 and Algorithm 2.6.

Remark 3.9 The bound $d^{2}+d$ in Proposition 3.7 is closely related to the intrinsic polarization in Proposition 2.8. If $\Gamma$ consists of an affine Dynkin diagram of degree $d$ and a single $n$-section, one has $h_{\Gamma}^{2}<2(d / n)^{2}+2(d / n)$. By the obvious monotonicity of $h_{\Gamma}^{2}$, if $D \geqslant d^{2}+d$, any (sub-)geometric graph containing an affine Dynkin diagram of degree $d$ is parabolic. (This conclusion can as well be derived from the proof of Proposition 3.7.)

\subsection{Proof of Theorem 1.5}

If the Fano graph Fn $X$ is hyperbolic, it contains a pencil $\Gamma$. There are but finitely many parabolic graphs satisfying (3.3) and (3.4), each of them has finitely many hyperbolic 1 -vertex extensions, and each extension is geometric for finitely many values of $D$ by Proposition 2.8. Thus, for $D \gg 0$, the graph Fn $X$ is either elliptic, and then $\mid$ Fn $X \mid \leqslant 19$ by (2.1), or parabolic. In the latter case, the bound $M_{\mathbb{C}}(D)$ on $|\mathrm{Fn} X|$ is given by Corollary 3.8 .

Remark 3.10 The precise lower bound on $D$ in Theorem 1.5 is rather high: the conclusion holds to full extent for $D \geqslant 621$. There exists a one-vertex extension of the affine Dynkin diagram $\widetilde{\mathbf{E}}_{8}$ (viz. $\widetilde{\mathbf{E}}_{8}$ itself and one simple section, cf. Fig. 1 in Sect. 4.1) which is geometric for all $D \leqslant 620$.

Remark 3.11 Corollary 3.8 shows also that, for each $D \geqslant 6$, there exists a smooth $2 D$-polarized $K 3$-surface $X$ with Fn $X$ parabolic and $|\mathrm{Fn} X|=21$ : namely, one can have Fn $X \cong 7 \widetilde{\mathbf{A}}_{2}$ or $6 \widetilde{\mathbf{A}}_{2}+3 \mathbf{A}_{1}$.

\subsection{Proof of Theorem 1.6}

In view of Theorem 1.5 , the bound $\left|\mathrm{Fn}_{\mathbb{R}} X\right| \leqslant 21$ and the values of $D$ for which it is sharp follow immediately from Theorem 2.7 and Corollary 3.4 (see Table 2, where real configurations are marked). For the lower bound $M_{\mathbb{R}}(D) \geqslant 19$ one can use a pencil $\Gamma \cong 6 \widetilde{\mathbf{A}}_{2}+\mathbf{A}_{1}$ or $2 \widetilde{\mathbf{E}}_{8}+\mathbf{A}_{1}$. The values of $D \gg 0$ for which $M_{\mathbb{R}}(D)=20$ are given by the classification of pencils of size 20 (using Remark 3.5): the complete list, which is too long, is found in [4].

\section{The Taxonomy of Hyperbolic Graphs}

In [5] and [2], the Fano graphs of spatial quartics are subdivided into triangular (all large graphs), quadrangular, and quadrangle free. In this paper, we adopt a more consistent 
taxonomy based on the type of a minimal fiber (see Definition 4.1): this approach allows a more refined classification and stronger bounds.

Till the rest of the paper, we consider hyperbolic graphs only. (Some of the statements do not make use of this assumption, merely asserting that a certain linear combination of $h$ and lines would have to be a bad vector in the sense of Definition 2.1. However, we do not single out these statements, as from our point of view graphs with $\sigma_{+}>1$ have no geometric meaning.) In the proofs, we implicitly refer to Corollary 3.8 or, if necessary, more refined classification of the parabolic graphs of a given type based directly on Proposition 3.7 (see also [4]).

\subsection{The Taxonomy}

Recall that any hyperbolic graph $\Gamma$ contains a connected parabolic subgraph: this obvious statement is part of the classification of elliptic and parabolic graphs.

Definition 4.1 A minimal fiber in a hyperbolic or parabolic graph $\Gamma$ is a connected parabolic subgraph (affine Dynkin diagram) $\Sigma \subset \Gamma$ minimal with respect to the following lexicographic order: we compare the Milnor numbers and, in the case of equal Milnor number $\mu$, let $\widetilde{\mathbf{A}}_{\mu}<\widetilde{\mathbf{D}}_{\mu}<\widetilde{\mathbf{E}}_{\mu}$. The graph $\Gamma$ itself is referred to as a $\Sigma$-graph, where $\Sigma$ is the (obviously well-defined) common isomorphism type of the minimal fibers in $\Gamma$. A minimal pencil in $\Gamma$ is any pencil of the form $\mathcal{P}(\Sigma) \subset \Gamma$, where $\Sigma \subset \Gamma$ is a minimal fiber. This terminology also applies to configurations $(S, h)$, according to the Fano graph $\operatorname{Fn}(S, h)$.

The $\widetilde{\mathbf{A}}_{2-}, \widetilde{\mathbf{A}}_{3^{-}}$, and $\widetilde{\mathbf{A}}_{4}$-graphs/configurations are alternatively called triangular, quadrangular, and pentagonal, respectively; these graphs $\Gamma$ are characterized by the property that girth $(\Gamma)=3,4$, and 5 , respectively.

The $\widetilde{\mathbf{D}}_{4}$-graphs/configurations are called astral; such graphs $\Gamma$ are characterized by the property that girth $(\Gamma) \geqslant 6$ and $\Gamma$ has a vertex of valency at least four. Since $\widetilde{\mathbf{D}}_{4}$ is the only affine Dynkin diagram with a vertex of valency 4 , any $\Sigma$-graph $\Gamma$ with $\mu(\Sigma) \geqslant 5$ is locally elliptic, i.e., one has val $v \leqslant 3$ for any vertex $v \in \Gamma$. Analyzing such affine Dynkin diagrams $\Sigma$ one by one, we easily arrive at the following bound on the number and positions of the sections of a minimal pencil.

Proposition 4.2 Let $\Sigma \subset \Gamma$ be a minimal fiber, and assume that $\mu(\Sigma) \geqslant 5$. Then, the pencil $\mathcal{P}(\Sigma)$ has at most three parabolic fibers and its sections are as shown in Fig. 1. Furthermore, all sections are simple and, unless $\Sigma \cong \widetilde{\mathbf{A}}_{5}$, pairwise disjoint. If $\Sigma \cong \widetilde{\mathbf{A}}_{5}$, two sections may intersect only if they are adjacent to opposite corners of the hexagon $\Sigma$.

Remark 4.3 Certainly, Fig. 1 shows maximal sets of sections. If $\Sigma \cong \widetilde{\mathbf{A}}_{7}$, the condition is that the "distance" between the two sections in the octagon $\Sigma$ must be at least 3 (as otherwise the graph would contain $\widetilde{\mathbf{D}}_{5}$ or $\widetilde{\mathbf{D}}_{6}$ ); hence, there are two distinct maximal sets. 

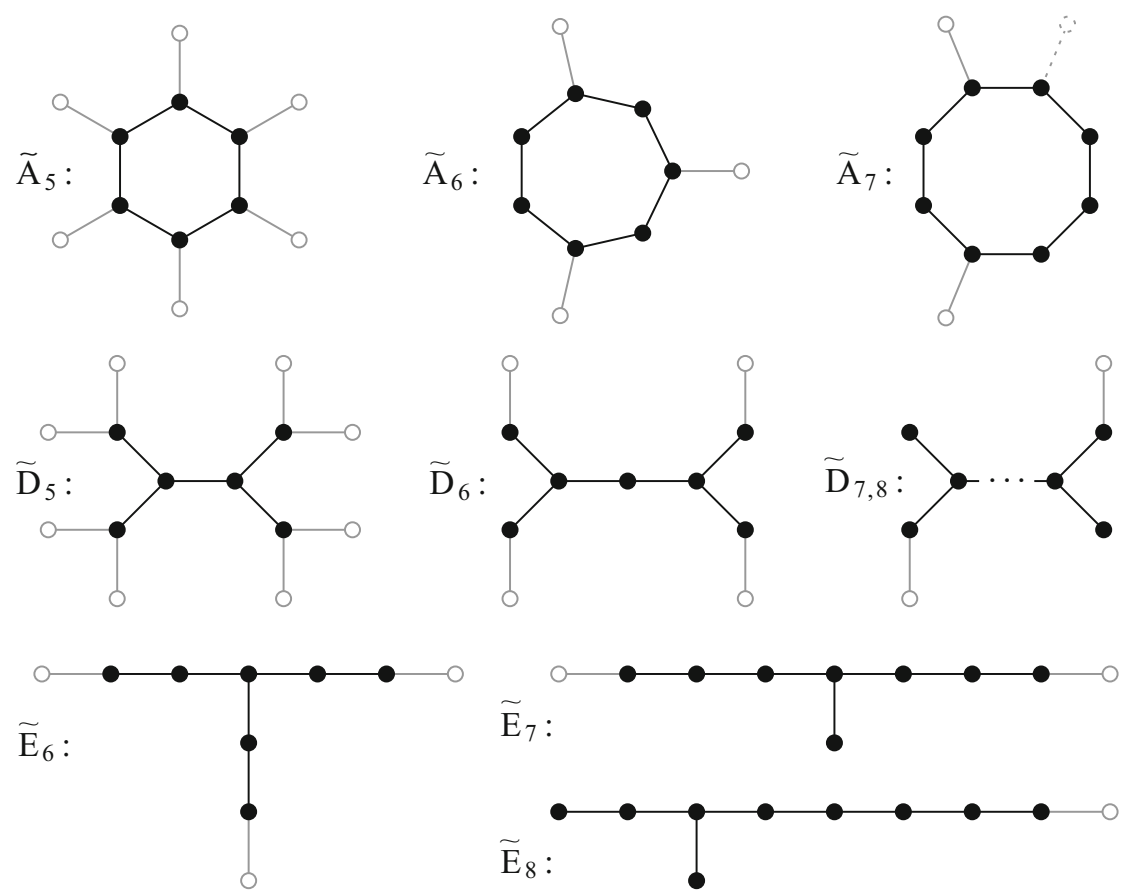

Fig. 1 Sections (white) at a minimal fiber (black) (see Proposition 4.2)

\subsection{Locally Elliptic Graphs}

In view of Theorems 1.5 and 1.6, most interesting are the configurations with twenty or more lines. In the locally elliptic case, one can easily list such configurations. (If $D$ is small, the number of configurations may be huge and we confine ourselves to those close to maximal.) The results are collected in the several statements below. In Theorem 4.6, we skip the values of $D$ for which $\bar{M}(D)=20$, as they are too erratic; these values, as well as a complete description of all graphs, are found in [4].

Theorem 4.4 (see Sect. 4.4) Let X be a smooth 2D-polarized K3-surface, and assume that $\mathrm{Fn} X$ is a hyperbolic $\widetilde{\mathbf{A}}_{5}$-graph. Then, with one exception $\mathrm{Fn} X \cong \Lambda_{25}$ shown in Table 3, one has a sharp bound $|\mathrm{Fn} X| \leqslant \bar{M}$, where $\bar{M}:=\bar{M}(D)$ is as follows:

$$
\begin{array}{cccccccc}
D & 2 . .12 & 13 & 14 & 15 & 16 . .20,22 \text { else } \\
\bar{M}: & 24 & 22 & 23 & 21 & 20 & \leqslant 19
\end{array}
$$

The notation in Table 3 is similar to Table 1 (see Sect. 1.3), with most fields skipped.

Theorem 4.5 (see Sect. 4.4) Let X be a smooth 2D-polarized K3-surface, and assume that $\mathrm{Fn} X$ is a hyperbolic $\widetilde{\mathbf{D}}_{5}$-graph. Then, with one exception $\mathrm{Fn} X \cong \Lambda_{28}$ shown in Table 3, one has a sharp bound $|\operatorname{Fn} X| \leqslant \bar{M}$, where $\bar{M}:=\bar{M}(D)$ is as follows: 
Table 3 Large locally elliptic graphs (see Theorems 4.4 and 4.5)

\begin{tabular}{llcllll}
\hline$D$ & $\Gamma$ & $\mid$ Aut $\Gamma \mid$ & det & $(r, c)$ & $\mid$ Aut $X \mid$ & \multicolumn{1}{c}{$T:=\mathcal{F}_{h}(\Gamma)^{\perp}$} \\
\hline 2 & $\Lambda_{25}$ & 144 & 780 & $(2,0)$ & 12 & {$[4,2,196]$} \\
4 & $\Lambda_{25}$ & 144 & 620 & $(2,0)$ & 12 & {$[4,2,156],[20,10,36]$} \\
6 & $\Lambda_{25}$ & 144 & 460 & $(1,0)$ & 12 & {$[20,10,28]$} \\
$10^{\dagger}$ & $\Lambda_{25}$ & 144 & 140 & $(1,0)$ & 12 & {$[12,2,12]$} \\
14 & $\Lambda_{28}{ }^{\ddagger}$ & 336 & $\underline{7}$ & $(1,0)$ & 336 & {$[2,1,4]$} \\
\hline
\end{tabular}

$$
\begin{array}{cccccc}
D: & 2 . .20 & 21,26,32 & 22,24 & 23,25,27,28,30,34,36 & \text { else } \\
\bar{M}: & 24 & 21 & 22 & 20 & \leqslant 19
\end{array}
$$

The extremal graph $\Lambda_{28}$ is 3-regular, 3-arc transitive, and distance regular; it is isomorphic to the so-called Coxeter graph (the only symmetric 3-regular graph on 28 vertices). The configuration has 21 minimal pencils, all of type $2 \widetilde{\mathbf{D}}_{5}+\widetilde{\mathbf{A}}_{7}$.

Theorem 4.6 (see Sect. 4.4) Let X be a smooth 2D-polarized K3-surface, and assume that Fn $X$ is a hyperbolic $\Sigma$-graph, $\mu(\Sigma) \geqslant 6$. Then $|\mathrm{Fn} X| \leqslant \bar{M}:=\bar{M}(D)$, where

- if $\Sigma \cong \widetilde{\mathbf{E}}_{8}$ and $D=309$, then $\bar{M}=21$;

- if $\Sigma \cong \widetilde{\mathbf{E}}_{7}$ and $D \in\{2$.. 99\}, then $\bar{M}=21$;

- if $\Sigma \cong \widetilde{\mathbf{A}}_{6}$ and $D \in\{18,21,22\}$, then $\bar{M}=21$;

- if $\Sigma \cong \widetilde{\mathbf{D}}_{6}$, then $\bar{M}$ is as follows:

$$
\begin{array}{ccc}
D: & 2 \ldots 10,12 \ldots 16,18 \ldots 28 & 11,17,29 . .34,36 . .38,40,50 \\
\bar{M}: & 22 & 21
\end{array}
$$

- if $\Sigma \cong \widetilde{\mathbf{E}}_{6}$, then $\bar{M}$ is as follows:

$$
\begin{array}{ccccc}
D: & 2 \ldots 38,40 \ldots 45 & 39 & 48 \ldots 50,64 \\
\bar{M}: & 22 & 24 & 21
\end{array}
$$

- in all other cases, $\bar{M} \leqslant 20$.

These bounds are sharp. (See [4] for the graphs and the values $\bar{M}(D)=20$.)

Addendum 4.7 (see Sect. 4.4) There are but six hyperbolic locally elliptic Fano graphs with 24 vertices:

- $\Lambda_{24}^{\mathrm{A}}$ : $\mid$ Aut $\Lambda_{24}^{\mathrm{A}} \mid=144,3$-regular, $\mathrm{gd}=\{2 . .12\}, \operatorname{gd}_{\mathbb{R}}=\{3 \ldots 6,8 . .12\}$;

- $\Lambda_{24}^{\mathrm{E}}: \mid$ Aut $\Lambda_{24}^{\mathrm{E}} \mid=72$, gd $=\operatorname{gd}_{\mathbb{R}}=\{39\}$;

- $\Lambda_{24}: \mid$ Aut $\Lambda_{24} \mid=48$, gd $=\{2 \ldots 20\}, \operatorname{gd}_{\mathbb{R}}=\{5,10,13,18\}$;

- $\Lambda_{24}^{\prime}: \mid$ Aut $\Lambda_{24}^{\prime} \mid=32$, gd $=\{2 \ldots 16\}, \operatorname{gd}_{\mathbb{R}}=\{7,12,15,16\}$;

- $\Lambda_{24}^{\prime \prime}: \mid$ Aut $\Lambda_{24}^{\prime \prime} \mid=12$, gd $=\{2 . .13\}, \operatorname{gd}_{\mathbb{R}}=\{5,7,12\}$;

- $\Lambda_{24}^{\prime \prime \prime}: \mid$ Aut $\Lambda_{24}^{\prime \prime \prime} \mid=8, \operatorname{gd}=\{14\}, \operatorname{gd}_{\mathbb{R}}=\varnothing$. 
As a consequence, for each $D \in\{2$.. 20\}, there exists a geometric hyperbolic locally elliptic configuration with 24 lines, and for each $D \in\{3 . .13,15,16\}$, there exists a real configuration with these properties (cf. the sharpness in Theorems 1.1 and 1.4).

In the subsequent sections we show that, if $D>16$ and $\mu(\Sigma) \leqslant 4$, the number of lines in geometric $\Sigma$-configurations $(S, h), h^{2}=2 D$, is maximized by parabolic graphs. Combining this observation with Theorems 4.4-4.6, we obtain precise sharp bounds on the number of lines.

Corollary 4.8 If $D>20$ (cf. Theorem 1.1), the maximum $M_{\mathbb{C}}(D)$ is as given by Theorem 1.5, with the exception of the following eight values: $M_{\mathbb{C}}(39)=24$ and $M_{\mathbb{C}}(D)=22$ for $D \in\{21,23,29,33,35,41,45\}$.

Corollary 4.9 If $D>16$ (cf. Theorem 1.4), one has

$$
M_{\mathbb{R}}(D)=\max \left\{M_{p}(D), M_{\mathrm{h}}(D)\right\},
$$

where $M_{p}(D)$ is the maximum over the parabolic graphs given by Theorem 1.6, and $M_{\mathrm{h}}(D)$ is the maximum over the hyperbolic graphs, which is as follows:

- $M_{\mathrm{h}}(D)=24$ if $D \in\{18,39\}$,

- $M_{\mathrm{h}}(D)=22$ if $D \in\{17,19,21 . .25,27,30,33,34,37,41,42,45\}$,

- $M_{\mathrm{h}}(D)=21$ if $D \in\{20,26,28,29,31,32,35,36,38,40,43,44,46$.. 99, 309\},

- $M_{\mathrm{h}}(D)=20$ if $D \in\{100 \ldots 308,342,396,565,576,601\}$,

and $M_{\mathrm{h}}(D) \leqslant 19$ for all other values $D>16$.

\subsection{Idea of the Proof}

The approach outlined in this section will be used in most proofs till the end of the paper.

We attempt to prove a bound $\mid$ Fn $X \mid \leqslant \bar{M}$ for graphs Fn $X$ satisfying certain conditions, subject to certain exceptions that are to be determined. To this end, we choose an appropriate pencil $\Gamma:=\mathcal{P}(\Sigma)$ with a distinguished fiber $\Sigma$ and try to estimate separately the two terms in (3.6). Typically, the bound given by the sum of the two estimates is too rough. Therefore, we choose appropriate thresholds $\bar{M}_{\Gamma}$ and $\bar{M}_{\text {sec }}$, so that $\bar{M}_{\Gamma}+\bar{M}_{\mathrm{sec}}=\bar{M}$, and try to classify, separately, the following two types of geometric graphs:

- pencils $\Gamma \supset \Sigma$ of size $|\Gamma|>\bar{M}_{\Gamma}$ with at least a few sections without which the count $\mid$ Fn $X \mid>\bar{M}$ is not reachable (see Sect. A.2), and

- section sets $\sec \Gamma$ (in the presence of just the single distinguished fiber $\Sigma$ ) of size $|\sec \Gamma|>\bar{M}_{\text {sec }}$ (see Sect. A.5).

The thresholds are chosen so that, on the one hand, the computation is feasible and, on the other hand, the partial configurations $S$ obtained have maximal or close to maximal rank. In the former case, $\mathrm{rk} S=20$, all geometric extensions of $S$ are found by Algorithm 2.6; in the latter case, we either add a few (usually, up to two) more lines to increase the rank to 20 or prove that $S$ has no further extensions.

The graphs Fn $X$ with $\mid$ Fn $X \mid>\bar{M}$ are found in the process of the classification, and all others satisfy the inequality $|\mathrm{Fn} X| \leqslant \bar{M}_{\Gamma}+\bar{M}_{\mathrm{sec}}=\bar{M}$ given by (3.6). 


\subsection{Proof of Theorems 4.4-4.6 and Addendum 4.7}

Given a configuration $S$ as in the statements, fix a minimal pencil $\Gamma:=\mathcal{P}(\Sigma) \subset$ Fn $S$. By Proposition 4.2, this pencil has at most three parabolic fibers, hence $|\Gamma| \leqslant 21$ by (3.3), and at most eight sections; thus, by (3.6), we have a bound $|\mathrm{Fn} S| \leqslant 29$, which holds in any degree and over any field (including positive characteristic).

For the more precise bounds as in the statements, we classify the configurations, running the modified version (see Sect. A.3) of the algorithm of Sect. A.2. For Theorem 4.6, we take $\bar{M}=19$, obtaining all graphs with 20 or more vertices. For Theorems 4.4, 4.5 and Addendum 4.7, in order to avoid too large output, we choose $19 \leqslant \bar{M} \leqslant 23$ close to the expected maximum, depending on $D$. The other thresholds used are $\bar{M}_{\Gamma}=\bar{M}-\bar{M}_{\mathrm{sec}}$ and $\bar{M}_{\mathrm{sec}}=\max |\sec \Gamma|$, where the latter maximum, depending on $\Sigma$ and $D$, is computed using Proposition 2.8. (If $\Sigma \cong \mathbf{A}_{5}$, we need to take into account the possible intersections of pairs of sections adjacent to opposite corner; up to automorphism, this results in 23 sets of sections.) A few technical observations (in terms of the coordinates, see Convention 3.6) reduce the number of candidates:

- $|\bar{s}| \leqslant 3$ for each $s \in \sec \Gamma$ (as the graph is locally elliptic);

- $\left|\bar{s}^{\prime} \cap \bar{s}^{\prime \prime}\right| \leqslant 1$ for $s^{\prime} \neq s^{\prime \prime} \in \sec \Gamma$ (as the graph is quadrangle free);

- $|\bar{s}| \leqslant 2$ for each $s \in \sec \Gamma$, if $\Sigma \cong \widetilde{\mathbf{D}}_{7}$ (no subgraphs isomorphic to $\widetilde{\mathbf{D}}_{6}$ );

- $|\bar{s}| \leqslant 1$ for each $s \in \sec \Gamma$, if $\Sigma \cong \widetilde{\mathbf{D}}_{8}$ (no subgraphs isomorphic to $\widetilde{\mathbf{E}}_{7}$ ).

Finally, the defining property $\mathfrak{P}$ is that $S$ should be a $\Sigma$-configuration: since we always have $\Sigma \subset$ Fn $S$, this property is obviously hereditary.

\section{Other Pencils with Disjoint Sections}

There are two other (unrelated) classes of pencils in Fano graphs whose sections are a priori known to be disjoint: astral configurations and Fano graphs of special octics. At the end of this section, in Sect. 5.5, we consider also the toy problem of counting lines in hyperelliptic models of degree $2 D \geqslant 6$.

\subsection{Astral Configurations}

Recall that astral are the $\widetilde{\mathbf{D}}_{4}$-graphs. These graphs $\Gamma$ are characterized by the property that $\operatorname{girth}(\Gamma) \geqslant 6$ and max val $v \geqslant 4, v \in \Gamma$.

Theorem 5.1 (see Sect. 5.2) Let X be a smooth 2D-polarized K3-surface, and assume that the Fano graph Fn $X$ is astral. Let $\bar{M}:=\bar{M}(D)$ be as in Table 4 or as follows:

$$
\begin{array}{cccccccc}
D: & 3,5,9 & 11,13 & 15 & 16 & \{17 \bmod 6\} & \{18 \bmod 2\} & \{19,21 \bmod 6\} \\
\bar{M}: & 24 & 22 & 20 & 21 & 18 & 20 & 19
\end{array}
$$

Then, with the exceptions listed in Table 4, one has a sharp bound $|\mathrm{Fn} X| \leqslant \bar{M}$.

The graph $\Delta_{32}^{\prime}$ in Table 4 is 4-regular, 3-arc transitive, distance regular, and bipartite. According to D. Pasechnik (private communication), this is the graph of points and 
Table 4 Extremal astral configurations (see Theorem 5.1)

\begin{tabular}{llllllll}
\hline$D$ & $\bar{M}$ & $\Gamma$ & $\mid$ Aut $\Gamma \mid$ & $\operatorname{det}$ & $(r, c)$ & $\mid$ Aut $X \mid$ & $T:=\mathcal{F}_{h}(\Gamma)^{\perp}$ \\
\hline 2 & 26 & $\Delta_{27}^{\prime}$ & 72 & 300 & $(2,0)$ & 12 & {$[4,2,76]$} \\
4 & 26 & $\Delta_{28}^{\prime}$ & 576 & 44 & $(1,0)$ & 96 & {$[4,2,12]$} \\
& & $\Delta_{27}^{\prime}$ & 72 & 204 & $(0,1)$ & 12 & {$[12,6,20]$} \\
& & $\Delta_{27}^{\prime \prime}$ & 72 & 207 & $(1,1)$ & 18 & {$[6,3,36],[12,3,18]^{*}$} \\
6 & 26 & $\Delta_{28}^{\prime}$ & 576 & 28 & $(1,0)$ & 96 & {$[4,2,8]$} \\
7 & 24 & $\Delta_{27}^{\prime \prime}$ & 72 & 99 & $(1,0)$ & 18 & {$[6,3,18]$} \\
8 & 24 & $\Delta_{32}^{\prime}$ & 2304 & 12 & $(1,0)$ & 1152 & {$[4,2,4]$} \\
& & $\Delta_{26}$ & 24 & 60 & $(1,0)$ & 6 & {$[4,2,16]$} \\
& & $\Delta_{25}^{\prime \prime}$ & 16 & 60 & $(1,0)$ & 4 & {$[4,2,16]$} \\
$10^{\dagger}$ & 24 & $\Delta_{25}^{\prime}$ & 24 & 96 & $(0,1)$ & 6 & {$[4,0,24]$} \\
12 & 23 & $\Delta_{24}^{\prime}$ & 1152 & & $\operatorname{dim}=1$ & & $\mathbf{U}(2) \oplus[4]$ \\
14 & 21 & $\Delta_{24}^{\prime}$ & 1152 & & $\operatorname{dim}=2$ & & $\mathbf{U}^{2}(2)$ \\
\hline
\end{tabular}

lines in the affine plane $\mathbb{F}_{4}^{2}$, with one pencil of parallel lines removed. The graph contains eight minimal pencils, all of type $4 \widetilde{\mathbf{D}}_{4}$.

Two other sufficiently symmetric graphs are $\Delta_{28}^{\prime}$ and $\Delta_{24}^{\prime}$, containing three and two minimal pencils, all of type $4 \widetilde{\mathbf{D}}_{4}$, respectively. Each graph $\Gamma$ is bipartite, the bicomponents $C_{1}, C_{2}$ are orbits of Aut $\Gamma$, and the action of Aut $\Gamma$ on $C_{1} \times C_{2}$ has two orbits, distinguished by whether vertices are adjacent or not.

The bipartite graphs $\Delta_{28}^{\prime}, \Delta_{27}^{\prime}$, and $\Delta_{24}^{\prime}$ are subgraphs of $\Delta_{32}^{\prime}$.

\subsection{Proof of Theorem 5.1}

Consider an astral configuration $S$, fix a minimal fiber $\Sigma \subset$ Fn $X$ of type $\widetilde{\mathbf{D}}_{4}$, and let $\Gamma:=\mathcal{P}(\Sigma)$ : this pencil may have parabolic fibers of type $\widetilde{\mathbf{D}}_{4}$ or $\widetilde{\mathbf{A}}_{5}$ and elliptic fibers of type $\mathbf{A}_{p}, p \leqslant 4$. Since we assume that girth $(\mathrm{Fn} S) \geqslant 6$, all sections of $\Gamma$ are pairwise disjoint, and their number is bounded by Proposition 2.8 as follows:

$$
\begin{array}{rccccccccccccc}
D & = & 2,4,6,8 & 3,5,7 & 9 & 10 & 11 & 12 \ldots 14 & 15,16 & 17 & . .20 & 21 \ldots 34 & \text { else } \\
|\sec \Gamma| \leqslant & 12 & 11 & 9 & 8 & 6 & 4 & 3 & 2 & 1 & 0
\end{array}
$$

(Strictly speaking, for large values of $D$ these bounds use an additional assumption that the pencil admits at least one extra line $l \in \Gamma \backslash \Sigma$.)

To simplify the further computation, we eliminate several large sets of sections. The following lemma is proved in Sect. A.8, after the necessary terminology is developed.

Lemma 5.2 (see Sect. A.8) In the settings of the theorem, one has:

(1) if $|\sec \Gamma|=12$, then $|\mathrm{Fn} S| \leqslant 24$ or $\mathrm{Fn} S \cong \Delta_{27}^{\prime}(D=2,4)$ or $\Delta_{32}^{\prime}(D=8)$;

(2) if $D=5$ and $|\sec \Gamma| \geqslant 11$, then $|\mathrm{Fn} S| \leqslant 22$. 
There remains to run the algorithm of Sect. A.3 (using a few further restrictions on the fibers of $\Gamma$, which are also stated in Sect. A.8) and list geometric configurations $S$ satisfying the inequality $|\mathrm{Fn} S|>\bar{M}$, where the threshold $\bar{M}$ is as in the statement of the theorem and $\bar{M}_{\text {sec }}$ is given by the table above, with 11 reduced down to 10 for $D=5$ and 12 reduced down to 11 , both due to Lemma 5.2.

\subsection{Geometry of Special Octics}

In this section, we assume $D=4$. Recall that an octic $X \subset \mathbb{P}^{5}$ is special if and only if its Néron-Severi lattice has a cubic pencil as in Definition 2.1(3), i.e., a class $e \in N S(X)$ such that $e^{2}=0$ and $e \cdot h=3$.

Let $l \in$ Fn $X$ be a line. The determinant of the lattice spanned by $h, l$, and $e$ is $-8 t^{2}+6 t+18$, where $t:=l \cdot e$; hence, this lattice is hyperbolic if and only if $l \cdot e=0, \pm 1$. If $l \cdot e=-1$, then the difference $e-l$ is a quadric pencil as in Definition 2.1(2). Thus, we conclude that

$$
l \cdot e=0 \text { or } 1 \text { for any line } l \in \mathrm{Fn} X
$$

Similarly, if a cubic pencil $e \in N S(X)$ exists, it is unique. Indeed, if there were two such classes $e_{1} \neq e_{2}$, the requirement that $N S(X)$ should be hyperbolic would imply that $e_{1} \cdot e_{2}=1$ or 2 . In the former case, $e_{1}-e_{2}$ would be an exceptional divisor as in Definition 2.1(1); in the latter, $h-e_{1}-e_{2}$ would be a quadric pencil as in Definition 2.1(2). Thus, below we denote this unique class by $e_{X} \in N S(X)$ and refer to it as the cubic pencil in $X$. As an immediate consequence, the graph Fn $X$ splits canonically into two sets

$$
C_{n}=C_{n}(X):=\left\{l \in \mathrm{Fn} X \mid l \cdot e_{X}=n\right\}, \quad n=0,1 .
$$

Definition 5.3 A biquadrangle in a graph $\Gamma$ is a complete bipartite subgraph $K_{2,3}$. In other words, a biquadrangle consists of a quadrangle (type $\widetilde{\mathbf{A}}_{3}$ fiber) $\Sigma=\left\{l_{1}, l_{2}, l_{3}, l_{4}\right\}$ and a bisection $s_{13}$ adjacent to two opposite corners $l_{1}, l_{3} \in \Sigma$.

Definition 5.4 A bipartite graph $\Gamma$ is called principal if it has a bicomponent, also called principal, containing all vertices of valency greater than two.

Proposition 5.5 Let $X \subset \mathbb{P}^{5}$ be a smooth octic. If $X$ is special, then either

(1) $\operatorname{girth}(\operatorname{Fn} X)=3$, or

(2) girth $(\mathrm{Fn} X)=4$ and $\mathrm{Fn} X$ has a biquadrangle (then, Fn $X$ is bipartite), or

(3) $\operatorname{girth}(\operatorname{Fn} X) \geqslant 6$ and $C_{1}(X)$ is a principal bicomponent of $\operatorname{Fn} X$.

Conversely, if Fn $X$ is as in items (1) or (2) above, then $X$ is special.

Proof Assume that $X$ is special and let $e:=e_{X}$.

Consider two lines $l_{1}, l_{2} \in \mathrm{Fn} X, l_{1} \cdot l_{2}=1$. If $l_{1} \cdot e=l_{2} \cdot e=0$, then the class $l_{3}:=e-l_{1}-l_{2}$ is also a line, and $\left\{l_{1}, l_{2}, l_{3}\right\}$ is a triangle. Conversely, if $\left\{l_{1}, l_{2}, l_{3}\right\}$ is a triangle, then the sum $e:=l_{1}+l_{2}+l_{3}$ is a cubic pencil and $X$ is special. 
In the rest of the proof, we assume that girth $($ Fn $X) \geqslant 4$.

If $l_{1} \cdot e=l_{2} \cdot e=1$, then $l_{1}+l_{2}+2 e-h$ is an exceptional divisor, contradicting to the assumption that $X$ is smooth. Thus, $C_{0}$ and $C_{1}$ are a pair of distinguished complimentary bicomponents and Fn $X$ is bipartite. Since $h$ is fixed, in the relation

$$
h=e+l_{1}+l_{2}+l_{3}+l_{4}+s_{13},
$$

the presence of any five classes implies the presence of the sixth. (This relation holds whenever $l_{1}, l_{2}, l_{3}, l_{4}, s_{13}$ are as in Definition 5.3 and $l_{1}, l_{3} \in C_{0}$.) Hence, the following statements are equivalent:

- the graph Fn $X$ has a biquadrangle, consisting of a quadrangle $\left\{l_{1}, l_{2}, l_{3}, l_{4}\right\}$ and a bisection $s_{13}$ intersecting $l_{1}$ and $l_{3}$;

- $X$ is special and the graph Fn $X$ has a quadrangle $\left\{l_{1}, l_{2}, l_{3}, l_{4}\right\}$; here, we can assume that $l_{1}, l_{3} \in C_{0}$ and $l_{2}, l_{4} \in C_{1}$;

- $X$ is special and there is a vertex $l_{1} \in C_{0}$ such that val $l_{1}>2$, i.e., incident to at least three other vertices $l_{2}, l_{4}, s_{13}$ (and thus, $C_{1}$ is not principal).

These observations complete the proof.

Corollary 5.6 (of the proof and the uniqueness of $e_{X}$ ) In Proposition 5.5(2), one has val $v \leqslant 3$ for each vertex $v \in C_{0}$. In particular, each minimal pencil has exactly one bisection (cf. Definition 5.3).

Proposition 5.7 Assume that $X$ is special and $\operatorname{girth}(\operatorname{Fn} X) \geqslant 4$, so that the graph Fn $X=C_{0} \cup C_{1}$ is bipartite. Then the following statements hold:

(1) $\left|C_{1}\right| \leqslant 7$ or $\left|C_{1}\right|=9$; in the latter case, val $v=3$ for each vertex $v \in C_{0}$;

(2) $\left|C_{0}\right| \leqslant 12$ and, if $\left|C_{0}\right| \geqslant 9$, then val $v \leqslant 6$ for each vertex $v \in C_{1}$.

Proof For Statement (1), it suffices to observe that any nine lines $l_{1}, \ldots, l_{9} \in C_{1}$ are subject to the relation $3 h=l_{1}+\ldots+l_{9}+5 e_{X}$.

The bound $\left|C_{0}\right| \leqslant 12$ follows from the fact that each line $l \in C_{0}$ is a component of a type $\widetilde{\mathbf{A}}_{1}$ (or $\widetilde{\mathbf{A}}_{1}^{*}$ ) fiber of the elliptic pencil $\pi: X \rightarrow \mathbb{P}^{1}$ defined by $e_{X}$ (the other component of this fiber being the conic $e_{X}-l$ ). The valency bound is mainly given by the inertia index of the lattice; in the border case $C_{0}=\left\{l_{1}, \ldots, l_{9}\right\}$ and a line $l \in C_{1}$ intersecting $l_{1}, \ldots, l_{7}$, the class

$$
l_{1}+\cdots+l_{7}-l_{8}-l_{9}+3 l-h
$$

is an exceptional divisor as in Definition 2.1(1). (In fact, the valency bound does not use the assumption that $X$ should be special.)

\subsection{Proof of Theorem 1.3}

In view of Proposition 5.5, there only remains to prove the bounds on the number of lines in a special octic $X$. 
Fig. 2 The configuration $\Theta_{21}$ (see Theorem 1.3)

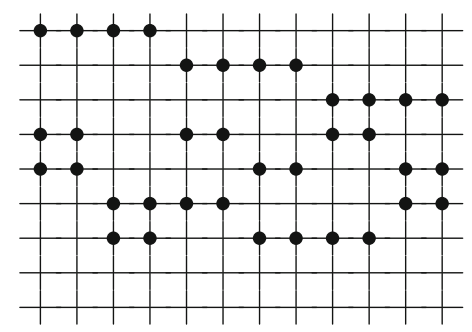

If girth $($ Fn $X)=3$, we pick a triangle $\Sigma \subset$ Fn $X$ and consider $\Gamma:=\mathcal{P}(\Sigma)$. The sections of $\Gamma$ are pairwise disjoint by Proposition 2.11, and Proposition 2.8 yields $|\sec \Gamma| \leqslant 9$; hence, $\mid$ Fn $X \mid \leqslant 33$, as stated. For the more refined statement, we run the algorithm of Sect. A.3 and list all configurations with more than $\bar{M}:=26$ lines.

Assume that girth(Fn $X) \geqslant 4$ and, hence, Fn $X=C_{0} \cup C_{1}$ is bipartite. By Proposition 5.7, we have either $\mid$ Fn $X \mid \leqslant 19$ or $\left|C_{1}\right|=9$ and $\left|C_{0}\right| \geqslant 11$; in the latter case, $\mid$ Fn $X \mid=20$ or 21 and, since $C_{1}$ is not a principal bicomponent, Fn $X$ has a biquadrangle by Proposition 5.5. For the refined classification, we run an algorithm similar to Sect. A.3, using a "pencil" $C_{0}=\left\{l_{1}, \ldots, l_{n}\right\}, n=11,12$, and adding "sections" $s \in C_{1}$ (regarded as subsets of $C_{0}$, cf. Convention 3.6). To form a biquadrangle, we start with a triple of sections $s_{1}, s_{2}, s_{3}$ such that

$$
\bar{s}_{1} \cap \bar{s}_{2}=\bar{s}_{2} \cap \bar{s}_{3}=\bar{s}_{3} \cap \bar{s}_{1}=\left\{l_{1}, l_{2}\right\} ;
$$

there are but three such triples. Then, at most three more sections can be added increasing the rank of the lattice, and we arrive at the dichotomy $\mid$ Fn $X \mid \leqslant 19$ or Fn $X \cong \Theta_{21}$ (see Fig. 2).

Remark 5.8 The computation at the end of the proof can be taken to a more combinatorial level if one takes into account the intrinsic structure on the set $C_{0}$ of size 12 or 11 arising from the imprimitive embedding $\mathbb{Z} C_{0}+\mathbb{Z} h+\mathbb{Z} c_{X} \hookrightarrow \mathbf{L}$ (cf. pencils of type $(0,12)$ and $(0,11)$ in $[5])$. We leave this exercise to the reader.

One can use Propositions 5.5 and 5.7 to take the classification one step further and find the extremal configurations that are neither triangular nor quadrangular. For such a graph $\Gamma=C_{0} \cup C_{1}$, we have $|\Gamma|<19$ unless $\left|C_{0}\right|=12$ and $\left|C_{1}\right|=7$. This time, the class $c_{X}$ does not need to belong to $\mathcal{F}_{h}(X)$; hence, we replace this lattice with the primitive hull of $\mathcal{F}_{h}(X)+\mathbb{Z} c_{X}$. The result of the straightforward computation using Sect. A.3 is as follows.

Proposition 5.9 Let $X \subset \mathbb{P}^{5}$ be a smooth special octic such that girth(Fn $\left.X\right) \geqslant 5$. Then Fn $X \cong \Delta_{19}^{\prime}, \Delta_{19}^{\prime \prime}$ (see Fig. 3) or $\mid$ Fn $X \mid \leqslant 18$. The extremal Fano graphs $\Delta_{19}^{\prime}$, $\Delta_{19}^{\prime \prime}$ can also be realized by smooth triquadrics.

\subsection{Hyperelliptic Models}

A hyperelliptic model of degree $2 D$ of a $K 3$-surface $X$ is a two-to-one map $X \rightarrow$ $Y \hookrightarrow \mathbb{P}^{D+1}$ splitting through a rational surface $Y$. Due to [14], a degree $2 D$ model 

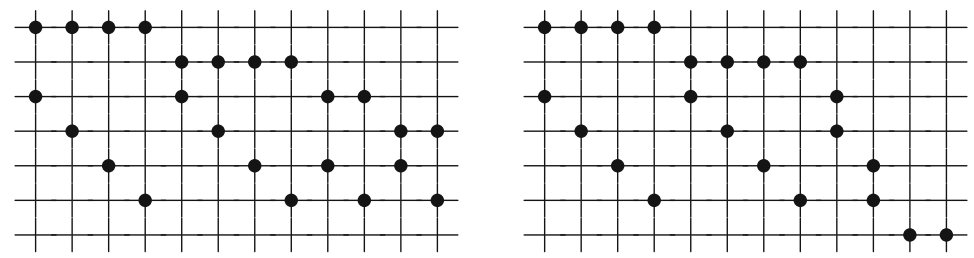

Fig. 3 The configurations $\Delta_{19}^{\prime}$ and $\Delta_{19}^{\prime \prime}$ (see Proposition 5.9)

$X \rightarrow \mathbb{P}^{D+1}$ defined by a class $h \in N S(X)$ is hyperelliptic if and only if one of the following conditions holds:

(1) $D=1$ : any model $X \rightarrow \mathbb{P}^{2}$ is hyperelliptic;

(2) $D=4$ and $h$ is divisible by 2 in $N S(X)$ : the model is the composition of a double covering $X \rightarrow \mathbb{P}^{2}$ and the Veronese embedding $\mathbb{P}^{2} \hookrightarrow \mathbb{P}^{5}$;

(3) $D \geqslant 2$ and there is a quadric pencil $e \in N S(X)$ as in Definition 2.1(2): the rational surface $Y \subset \mathbb{P}^{D+1}$ is a scroll in this case (see also [13]).

A hyperelliptic model $X \rightarrow Y \hookrightarrow \mathbb{P}^{D+1}$ is considered smooth if so is its ramification locus in $Y$. A line in a hyperelliptic model is a rational curve $L \subset X$ that is mapped one-to-one onto a line in $\mathbb{P}^{D+1}$. Thus, arithmetically, a line can still be described as a class $l \in N S(X)$ such that $l^{2}=-2$ and $l \cdot h=1$.

We will treat the case $D=1$ in a subsequent paper: the conjectural bound for such models is 144 lines (see [3]).

In Case (2), where the model splits through the Veronese embedding, it has no lines, as $l \cdot h=0 \bmod 2$ for any $l \in N S(X)$.

Thus, we are left with Case (3), where the image $Y$ is a scroll. To study such models, we need to change the definition of the admissibility of a configuration $S$ (see Definition 2.1): the exceptional divisors as in (1) are still excluded, but this time we assume the presence of a quadric pencil $e \in S$ as in (2), requiring instead that there should be no class $c \in S$ such that

$\left(2^{\prime}\right) c^{2}=0$ and $c \cdot h=1$ (fixed component, see [10]).

Now, arguing as in Sect. 5.3 and using an obvious analogue of Proposition 2.8, we can easily prove the following statement.

Lemma 5.10 Consider a smooth hyperelliptic model $X \rightarrow \mathbb{P}^{D+1}, D \geqslant 2$, and let $e \in N S(X)$ be a quadric pencil. Then, for any line $l \in \mathrm{Fn} X$, we have $l \cdot e=0$ or 1 . Furthermore, if $D \geqslant 3$, the quadric pencil $e:=e_{X} \in N S(X)$ is unique.

If $D=2$, the obvious maximum of 48 lines (24 over each of the two rulings) is realized by a connected equilinear family containing, in particular, the discriminant minimizing singular surfaces (see [3]).

Thus, from now on, we assume that $D \geqslant 3$, and Lemma 5.10 gives us a natural splitting Fn $X=C_{0} \cup C_{1}$ as in (5.2). The set $C_{0}$ consists of the components of the reducible fibers of an elliptic pencil-the pullback of the ruling of $Y$; these fibers are of type $\widetilde{\mathbf{A}}_{1}$ (or $\widetilde{\mathbf{A}}_{1}^{*}$ ), we have $l^{\prime} \cdot l^{\prime \prime}=2$ for two lines $l^{\prime}, l^{\prime \prime}$ in the same fiber, and each line $l \in C_{1}$ intersects exactly one of $l^{\prime}, l^{\prime \prime}$. Further computation shows that the set $C_{1}$ is empty with the following few exceptions: 
- $D=3$ and $C_{1}=\left\{l^{\prime}, l^{\prime \prime}\right\}, l^{\prime} \cdot l^{\prime \prime}=1$ and $h=l^{\prime}+l^{\prime \prime}+2 e$;

- $D=4$ and $C_{1}=\left\{l^{\prime}, l^{\prime \prime}\right\}, l^{\prime} \cdot l^{\prime \prime}=0$ and $h=l^{\prime}+l^{\prime \prime}+3 e$;

- $D=6$ and $C_{1}=\{l\}$ so that $h=2 l+5 e$; hence, $C_{0}=\varnothing$.

Geometrically, $C_{1}$ projects to the exceptional section $E \subset Y$; if $D=6\left(E^{2}=-4\right)$, this section is a component of the ramification locus.

Theorem 5.11 Let $X \rightarrow \mathbb{P}^{D+1}$ be a hyperelliptic model, $D \geqslant 3$. Then, unless $D=6$ and $|\mathrm{Fn} X|=1$, we have $|\mathrm{Fn} X|=0 \bmod 2$ and $|\mathrm{Fn} X| \leqslant 24-4 \varepsilon$, where we let $\varepsilon:=D \bmod 2 \in\{0,1\}$. This bound is sharp.

Proof If $C_{1}=\varnothing$, the configuration consists of a single pencil $C_{0}$ and, by an obvious analogue of Proposition 3.7, the maximal number of lines depends on $D$ mod 2 only. Using Algorithm 2.6, we arrive at $|\operatorname{Fn} X| \leqslant 24-4 \varepsilon$. The other case $\left|C_{1}\right|=2$ is treated similarly: for each value $D=3,4$ and each cardinality $\left|C_{0}\right|$, there is a single candidate, which is analyzed using Algorithm 2.6.

\section{Pentagonal Configurations}

In the remaining three sections, we consider configurations with minimal pencils whose sections may intersect each other.

\subsection{Statements}

Recall that a graph $\Gamma$ is pentagonal if girth $(\Gamma)=5$. All large geometric pentagonal graphs are described by the following theorem.

Theorem 6.1 (see Sect. 6.3) Let X be a smooth 2D-polarized K3-surface, and assume that the Fano graph $\mathrm{Fn} X$ is pentagonal, i.e., girth $(\mathrm{Fn} X)=5$. Let $\bar{M}:=\bar{M}(D)$ be as in Table 5 or as follows:

$$
\begin{array}{cccc}
D: & \{10,11 \bmod 5\} & \{12 \bmod 5\} & \text { else } \\
\bar{M}: & 21 & 22 & 20
\end{array}
$$

Then, with the exceptions listed in Table 5, one has a sharp bound $|\mathrm{Fn} X| \leqslant \bar{M}$.

The notation used in Table 4 is similar to Table 1 (with some columns skipped); it is explained in Sect. 1.3. It is remarkable that the same graph $\Phi_{30}^{\prime}$ is a Fano graph of $2 D$-polarized $K 3$-surfaces for all $2 \leqslant D \leqslant 7$. The graph $\Phi_{30}^{\prime \prime}$ works for $2 \leqslant D \leqslant 5$; if $D=6$, this graph is still geometric, but not saturated (pencils acquire extra fibers and/or sections), and $\Phi_{36}^{\prime \prime} \supset \Phi_{30}^{\prime \prime}$ is its saturation.

The graph $\Phi_{36}^{\prime \prime}$ is 5-regular, 2-arc transitive, and distance regular; according to D. Pasechnik (private communication), it is the Sylvester graph. The graph contains 36 minimal pencils, all of type $4 \widetilde{\mathbf{A}}_{4}+\mathbf{A}_{1}$.

The graph $\Phi_{30}^{\prime}$ is 4-regular and 1-arc transitive; it contains six copies of $4 \widetilde{\mathbf{A}}_{4}$. 
Table 5 Extremal pentagonal configurations (see Theorem 6.1)

\begin{tabular}{lccccccl}
\hline$D$ & $\bar{M}$ & $\Gamma$ & $\mid$ Aut $\Gamma \mid$ & det & $(r, c)$ & $\mid$ Aut $X \mid$ & $T:=\mathcal{F}_{h}(\Gamma)^{\perp}$ \\
\hline 2 & 29 & $\Phi_{30}^{\prime}$ & 240 & 220 & $(2,0)$ & 60 & {$[4,2,56],[16,6,16]$} \\
& & $\Phi_{30}^{\prime \prime}$ & 40 & 255 & $(1,1)$ & 10 & {$[4,1,64]^{*},[16,1,16]$} \\
3 & 29 & $\Phi_{30}^{\prime}$ & 240 & 180 & $(1,0)$ & 60 & {$[14,4,14]$} \\
& & $\Phi_{30}^{\prime \prime}$ & 40 & 195 & $(1,0)$ & 10 & {$[14,1,14]$} \\
4 & 29 & $\Phi_{30}^{\prime}$ & 240 & 140 & $(1,1)$ & 60 & {$[12,2,12]$} \\
& & $\Phi_{30}^{\prime \prime}$ & 40 & 135 & $(1,1)$ & 10 & {$[12,3,12]$} \\
$5^{\dagger}$ & 28 & $\Phi_{30}^{\prime}$ & 240 & 100 & $(1,0)$ & 60 & {$[10,0,10]$} \\
& & $\Phi_{30}^{\prime \prime}$ & 40 & 75 & $(1,0)$ & 10 & {$[10,5,10]$} \\
& & $\Phi_{30}^{\prime \prime \prime}$ & 24 & 36 & $(1,0)$ & 12 & {$[4,2,10]$} \\
6 & 28 & $\Phi_{30}^{\prime}$ & 240 & 60 & $(1,0)$ & 60 & {$[8,2,8]$} \\
& & $\Phi_{36}^{\prime \prime}$ & 1440 & $\underline{15}$ & $(1,0)$ & 720 & {$[2,1,8]$} \\
7 & 26 & $\Phi_{30}^{\prime}$ & 240 & $\underline{20}$ & $(1,0)$ & 120 & {$[4,2,6]$} \\
8 & 24 & $\Phi_{25}$ & 80 & & $\operatorname{dim}=1$ & & $\mathbf{U}(5) \oplus[4]$ \\
9 & 23 & $\Phi_{25}^{\ddagger}$ & 80 & $\underline{15}$ & $\operatorname{dim}=1$ & & $\mathbf{U}(5) \oplus[2]$ \\
$10^{\dagger}$ & 21 & $\Phi_{25}^{\ddagger}$ & 80 & & $\operatorname{dim}=2$ & & $\mathbf{U}(5) \oplus \mathbf{U}$ \\
\hline
\end{tabular}

\subsection{Sections at a Fiber $\Sigma$ of Type $\tilde{A}_{4}$}

Consider a geometric pentagonal configuration $S$ and pick a distinguished pentagon $\Sigma:=\left\{l_{1}, \ldots, l_{5}\right\} \subset$ Fn $S$.

Convention 6.2 Here and below, we always assume that the $n$ lines $l_{1}, \ldots, l_{n}$ of a type $\widetilde{\mathbf{A}}_{n-1}$ fiber in a configuration are numbered cyclically, so that

$$
l_{i \pm n}=l_{i}, \quad l_{i} \cdot l_{j}=1 \text { if and only if } i-j= \pm 1 \bmod n .
$$

Thus, we regard the indices as elements of the cyclic group $\mathbb{Z} / n$.

Consider the pencil $\Gamma:=\mathcal{P}(\Sigma)$. Since Fn $S$ has no triangles or quadrangles, we immediately obtain the following lemma.

Lemma 6.3 For $\Sigma \subset \Gamma \subset$ Fn $S$ as above, the following statements hold:

- all sections of $\Gamma$ are simple;

- $\left(\sec l_{i}\right) *\left(\sec l_{j}\right)=0$ unless $i-j= \pm 2 \bmod 5$;

- $\left(\sec l_{i}\right) *\left(\sec l_{j}\right) \leqslant 1$ if $i-j= \pm 2 \bmod 5$.

In particular, $\Sigma$ is suitable for enumeration in the sense of Sect. A.5.

Lemma 6.4 For $\Sigma \subset \Gamma \subset$ Fn $S$ as above, one has the following bounds on $|\sec \Gamma|$ :

$$
\begin{array}{rlccccccccccccc}
D & = & 2,3 & 4 & 5 & 6 & 7 & 8 & 9,10 & 11,12 & 13 . .15 & 16 . .25 & \text { else } \\
|\sec \Gamma| & \leqslant & 16 & 17 & 18 & 15 & 10 & 7 & 5 & 3 & 2 & 1 & 0
\end{array}
$$


Furthermore, the following statements hold:

(1) if $2 \leqslant D \leqslant 4$ and $|\sec \Gamma| \geqslant 14$, then $|\operatorname{Fn} S| \leqslant 29$;

(2) if $D=5$ and $|\sec \Gamma| \geqslant 14$, then $\mathrm{Fn} S \cong \Phi_{30}^{\prime \prime \prime}$ or $|\mathrm{Fn} S| \leqslant 28$;

(3) if $D=6$ and $|\sec \Gamma| \geqslant 12$, then $\mathrm{Fn} S \cong \Phi_{36}^{\prime \prime}$ or $|\mathrm{Fn} S| \leqslant 28$;

(4) if $7 \leqslant D \leqslant 9$, then there is at most one pair of intersecting sections;

(5) if $D \geqslant 10$, then all sections of $\Gamma$ are pairwise disjoint.

Besides, if $D=5$, then val $l \in\{0 \ldots 3,5\}$ for each line $l \in \Sigma$.

Proof Since $\Sigma$ is suitable for enumeration, we can run the algorithm of Sect. A.5.

If $D \geqslant 7$, we can easily enumerate all sets of sections, obtaining, in particular,

Statements (4) and (5). (These lists are also used in Sect. 6.3.)

If $D \leqslant 6$, we only list the sets $\sec \Gamma$ with $|\sec \Gamma| \geqslant 14(|\sec \Gamma| \geqslant 12$ if $D=6)$. Then, Statements (1)-(3) are proved by applying Step 3 (see Sect. A.5) in order to classify large configurations. The technical details are explained in Sect. A.7.

The last statement is straightforward.

\subsection{Proof of Theorem 6.1}

Following the idea explained in Sect. 4.3, we can still use the algorithm of Sect. A.3 based on discrete sets of sections.

Let $\bar{M}$ be as in the statement of the theorem. If $D \geqslant 14$, there are relatively few sets of sections sec $\Gamma$ (see Lemma 6.4 and its proof), and we can use the algorithm directly, taking into account (if present) the one pair of sections that intersect.

If $D \leqslant 12$, we use the following trick. Let $\bar{M}_{\mathrm{sec}}=13\left(\bar{M}_{\mathrm{sec}}=11\right.$ if $\left.D=12\right)$. Then, by Lemma 6.4, it suffices to consider pencils $\Gamma$ with $|\Gamma|>\bar{M}_{\Gamma}:=\bar{M}-\bar{M}_{\text {sec }}$. Pick such a pencil $\Gamma$ and let $n_{i}:=\operatorname{val} l_{i}, i \in \mathbb{Z} / 5$, be a desired set of goals, such that

$$
\bar{M}-|\Gamma|<\sum_{i \in \mathbb{Z} / 5} n_{i} \leqslant \bar{M}_{\mathrm{sec}}
$$

(In addition, we can require that each pair $\left(n_{i}, n_{i+1}\right), i \in \mathbb{Z} / 5$, is realized by the valencies of a pair of adjacent lines of $\Sigma$ in a geometric configuration: this condition is easily checked as all sections involved are pairwise disjoint, see Lemma 6.3.) Let, further, $m:=\max \left\{n_{i}+n_{i+1} \mid i \in \mathbb{Z} / 5\right\}$, and consider the set

$$
\mathcal{N}\left(n_{i}\right):=\left\{(p, q) \mid p \geqslant q, p+q=m,(p, q)=\left(n_{i}, n_{i \pm 1}\right) \text { for some } i \in \mathbb{Z} / 5\right\}
$$

Then, denoting by $\mathcal{N}$ the union of $\mathcal{N}\left(n_{i}\right)$ over all sets of goals $\left(n_{i}\right)$ satisfying (6.1), we can assert that, in any geometric pentagonal extension $S \supset \mathcal{F}_{h}(\Gamma)$ such that $\mid$ Fn $X \mid>\bar{M}$, there is a pair $(p, q) \in \mathcal{N}$ such that $\Gamma$ has at least $(p+q)$ disjoint sections: up to automorphism of $\Sigma$, we can assume that val $l_{1} \geqslant p$ and val $l_{2} \geqslant q$.

If $D \in\{2 . .4,6\}$, the computation based on this observation completes the proof, as all configurations obtained by adding to the pencil $(p+q)$ disjoint sections as above have the maximal rank 20 and can be analyzed using Algorithm 2.6. 
If $D=5$, then, occasionally, we may need to add an extra section. Namely, let $\left(n_{i}\right)$ be a set of goals such that $2 m \leqslant \sum_{i} n_{i}$ : this is the case for all configurations of low rank. Then, a simple argument shows that we can reindex the lines so that $n_{1} \geqslant n_{2}$, $n_{1}+n_{2}=m$ is maximal, and $n_{3}>0$, i.e., $\Gamma$ has at least one section adjacent to $l_{3}$. Taking this extra section into account and allowing it to intersect one of those adjacent to $l_{1}$, we always obtain a configuration of the maximal rank 20 , and there remains to apply Algorithm 2.6.

\section{Quadrangular Configurations}

Recall that a graph $\Gamma$ and the corresponding configuration are quadrangular if $\operatorname{girth}(\Gamma)=4$, i.e., $\Gamma$ has no triangles, but it does contain a quadrangle.

\subsection{Statements}

In this section, we mostly ignore the case $D=2$, although the maximal cardinality of a triangle free configuration of lines in a spatial quartic remains an open problem (see the discussion in Sect. 7.5). The next statement is the principal result of the section.

Theorem 7.1 (see Sect. 7.4) Let $X \subset \mathbb{P}^{D+1}$ be a smooth $2 D$-polarized K3-surface, $D \geqslant 3$, and assume that the Fano graph $\mathrm{Fn} X$ is quadrangular, i.e., girth $(\operatorname{Fn} X)=4$. Then, with few exceptions, we have the following sharp bounds:

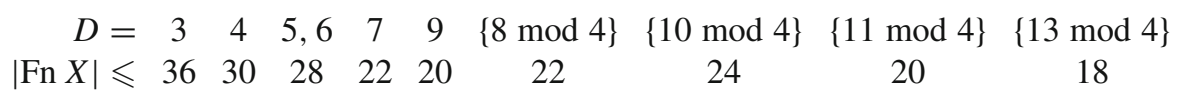

The exception is the case $D=4$, Fn $X \cong \Theta_{*}^{*}$ (see Table 1 and comments below).

Addendum 7.2 (see Sect. 7.4) The complete set $\{|\mathrm{Fn} X|\}$ of values taken by the line count of a quadrangular configuration is as follows:

- if $D=3$, then $\{|\mathrm{Fn} X|\}=\{4 . .36\}$;

- if $D=4$, then $\{|\mathrm{Fn} X|\}=\{4 . .30,32$.. 34, 36 $\}$.

According to Theorem 1.3, the exceptional Fano graphs $\Theta_{*}^{*}$ in Table 1 are realized by triquadrics only. The next statement is proved by a simple computation.

Addendum 7.3 There are inclusions $\Theta_{32}^{\prime} \subset \Theta_{34}^{\prime} \subset \Theta_{36}^{\prime}$ and, hence, respective specializations of the corresponding families of triquadrics. All other graphs $\Theta_{*}^{*}$ in Theorem 7.1 are maximal with respect to inclusion.

The graphs $\Theta_{36}^{\prime \prime}, \Theta_{32}^{\prime}, \Theta_{32}^{\prime \prime}, \Theta_{32}^{\prime \prime \prime}$, and $\Theta_{32}^{\mathrm{K}}$ are 6-regular and vertex transitive. The last one, $\Theta_{32}^{\mathrm{K}}$, is the famous Kummer configuration 16 $16_{6}$ it is 2-arc transitive, distance regular, and bipartite. The graphs $\Theta_{36}^{\prime}$ and $\Theta_{32}^{\mathrm{K}}$ can be represented by the discriminant minimizing singular $K 3$-surface $X([4,0,8]$ ) (see [3]). More details on these graphs and corresponding triquadrics are found in Table 1 (see also [4]). 


\subsection{Sections at a Fiber $\Sigma$ of Type $\tilde{A}_{3}$}

Till the end of this section, we consider a geometric quadrangular configuration $S$ and pick a quadrangle $\Sigma:=\left\{l_{1}, l_{2}, l_{3}, l_{4}\right\}$. We assume the lines ordered cyclically (see Convention 6.2) and, usually, so that

$$
\operatorname{val} l_{1} \geqslant \operatorname{val} l_{3}, \quad \operatorname{val} l_{1} \geqslant \operatorname{val} l_{2} \geqslant \operatorname{val} l_{4}, \quad \operatorname{val} l_{3} \geqslant \operatorname{val} l_{4} \text { if } \operatorname{val} l_{1}=\operatorname{val} l_{2}
$$

Consider also the pencil $\Gamma:=\mathcal{P}(\Sigma)$; since $k_{\Sigma}=l_{1}+l_{2}+l_{3}+l_{4}$ and $\operatorname{deg} \Gamma=4$, all fibers of $\Gamma$ are of types $\widetilde{\mathbf{A}}_{3}, \mathbf{A}_{2}$, or $\mathbf{A}_{1}$.

We subdivide quadrangular configurations according to whether they do or do not have a biquadrangle (see Definition 5.3).

Lemma 7.4 For $\Sigma \subset$ Fn $S$ as above, one has:

(1) each subgraph $\sec l_{i}, 1 \leqslant i \leqslant 4$, is discrete;

(2) $\sec l_{i} \cap \sec l_{j}=\varnothing$ whenever $j-i=1 \bmod 2$;

(3) if $D=3$, then $\left(\sec l_{i}\right) *\left(\sec l_{i \pm 1}\right) \leqslant 2$ and $\left(\sec l_{i}\right) *\left(\sec l_{i+2}\right) \leqslant 3$;

(4) if $D \geqslant 4$, then $\left(\sec l_{i}\right) *\left(\sec l_{j}\right) \leqslant 1$ for any pair $(i, j)$;

(5) if $D \geqslant 8$, then the subgraph sec $\Gamma$ is discrete.

If, in addition, $S$ has no biquadrangle, then also

(6) $\sec l_{i} \cap \sec l_{j}=\varnothing$ for any pair $1 \leqslant i<j \leqslant 4$;

(7) $\left(\sec l_{i}\right) *\left(\sec l_{i \pm 1}\right) \leqslant 1$ and $\left(\sec l_{i}\right) *\left(\sec l_{i+2}\right) \leqslant 2$.

(In view of Statement (4), the last restriction is meaningful only for $D=2,3$.)

Proof Most assertions are immediate from the assumption that $S$ should have no triangle or, for the last two statements, biquadrangle. Statements (3), (4), and (5) are proved by applying Proposition 2.8 to simple test configurations.

Lemma 7.5 If $S$ has no biquadrangle, one has the following bounds on $|\sec \Gamma|$ :

$$
\begin{array}{rlccccccccc}
D & = & 2,3 & 4 & 5 & 6 & 7,8 & 9,10 & 11 \ldots 16 & \text { else } \\
|\sec \Gamma| & \leqslant & 21 & 20 & 12 & 8 & 4 & 2 & 1 & 0
\end{array}
$$

Furthermore, the following statements hold:

- if $D=2,3$ and $|\sec \Gamma|=21$, then $|\mathrm{Fn} S|=34$ (a unique configuration);

- if $D=4$ and $|\sec \Gamma| \geqslant 15$, then either $|\mathrm{Fn} S| \leqslant 30$ or Fn $S$ is isomorphic to one of the exceptional graphs $\Theta_{36}^{\prime}, \Theta_{36}^{\prime \prime}, \Theta_{34}^{\prime}, \Theta_{32}^{\prime}, \Theta_{32}^{\prime \prime}, \Theta_{32}^{\prime \prime \prime}, \Theta_{32}^{\mathrm{K}}$.

Proof If $D \geqslant 4$, then, due to Lemma 7.4, the fiber $\Sigma$ is suitable for enumeration in the sense of Sect. A.5. Applying the algorithm of Sect. A.5 (in the order $l_{1}, l_{3}, l_{2}, l_{4}$ ), assuming (7.1) and taking for the defining property

$$
\mathfrak{P}: S \longmapsto S \text { has neither a triangle nor a biquadrangle, }
$$

we arrive at the statement of the lemma. 
Fig. 4 A biquadrangle for $D=3$

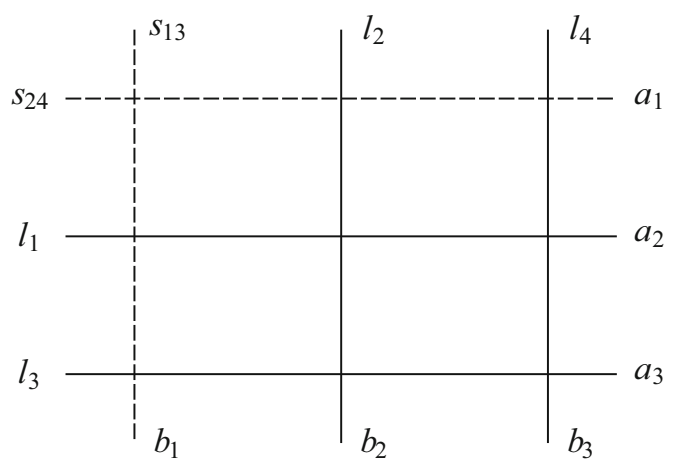

If $D=4$ and $|\sec \Gamma| \geqslant 15$, the configurations are classified using Step 3 of the algorithm (see Sect. A.5.4); we postpone the proof until Sect. A.6, after the algorithm is explained in more details.

If $D=2,3$, the situation is more complicated, see Lemma 7.4(7). However, large configurations can still be enumerated similar to Sect. A.5. Assuming that val $l_{1} \geqslant 6$ and val $l_{1}+\operatorname{val} l_{3} \geqslant 11$, see (7.1), we arrive at a unique configuration $S$ satisfying the inequality $|\sec \Gamma|>20$. This configuration has rank 20, contains 34 lines, and has no nontrivial $\mathfrak{P}$-geometric finite index extensions.

\subsection{Configurations with a Biquadrangle}

Assume that the pencil $\Gamma=\mathcal{P}(\Sigma)$ has a bisection $s_{13}$ intersecting $l_{1}$ and $l_{3}$. Using Proposition 2.8, one can show that $D \leqslant 4$ and, unless $D=2$, such a bisection is unique. We ignore the case $D=2$ (cf. Example 7.9), whereas the case $D=4$ was considered in Sect. 5.3, resulting in the bound $\mid$ Fn $S \mid \leqslant 21$ (see Theorem 1.3).

Lemma 7.6 Assume that $D=3$ and $\Gamma$ has a bisection $s_{13}$ intersecting $l_{1}$ and $l_{3}$. Then $\mid$ Fn $S \mid \leqslant 36$.

Proof It is immediate that $\Gamma$ has another bisection, viz.

$$
s_{24}:=h-l_{1}-l_{2}-l_{3}-l_{4}-s_{13},
$$

intersecting $l_{2}$ and $l_{4}$, so that $s_{13} \cdot s_{24}=1$, and any other line $l \in$ Fn $S$ intersects exactly one of $l_{1}, l_{2}, l_{3}, l_{4}, s_{13}, s_{24}$. Thus, renaming the lines to $a_{1}, a_{2}, a_{3}, b_{1}, b_{2}, b_{3}$ as in Fig. 4, we have

$$
|\mathrm{Fn} S|=\sum_{i=1}^{3} \operatorname{val} a_{i}+\sum_{j=1}^{3} \operatorname{val} b_{j}-12=\frac{1}{3} \sum_{i, j=1}^{3}\left(\operatorname{val} a_{i}+\operatorname{val} b_{j}\right)-12
$$

Since val $l \leqslant 9$ for each line $l \in \operatorname{Fn} S$ (see Proposition 2.10), we have $\mid$ Fn $S \mid \leqslant 42$. 
From now on, assume that $|\mathrm{Fn} S|>36$. Reindexing the lines (and choosing, if necessary, another quadrangle $\Sigma$ ), we can also assume that val $a_{1} \geqslant$ val $b_{1}$ and the pair $(i, j)=(1,1)$ maximizes the sum val $a_{i}+\operatorname{val} b_{j}, i, j=1,2,3$. By the count above, val $a_{1}=9$ and val $b_{1}=9$ or 8 ; hence, $|\Gamma|=\operatorname{val} a_{1}+\operatorname{val} b_{1}-2=16$ or 15 , respectively. In the former case, (7.1) implies val $l_{1} \geqslant 8$. In the latter case, we can order the lines so that val $l_{4} \leqslant$ val $l_{2} \leqslant 8$ and val $l_{3} \leqslant$ val $l_{1}$; then again val $l_{1} \geqslant 8$.

Now, we can run an algorithm similar to that of Sect. A.2, taking "triangle free" for the defining property $\mathfrak{P}$ and starting with a pencil $\Gamma$ of size $|\Gamma|=16$ or 15 and a pair of bisections $s_{13}, s_{24}$. Recall that each line in Fn $S$ intersects exactly one of the bisections $s_{13}, s_{24}$; together with the assumption that $S$ should be triangle free, this observation determines the pair of bisections uniquely up to Aut $\Gamma$. Furthermore, all other sections of $\Gamma$ are simple and disjoint from $s_{13}, s_{24}$. Thus, taking $l_{1}$ for $l$, at Steps 1 and 2 we add at least five disjoint sections $s_{i} \in \sec l$ (recall that val $l_{1} \geqslant 8$ ). Configurations of low rank (Step 3) are analyzed as described in Sect. A.4.2.

\subsection{Proof of Theorem 7.1 and Addendum 7.2}

Let $S:=\mathcal{F}_{h}(X)$ be as in the theorem. If $S$ has a biquadrangle, the statement is given by Lemma 7.6 and Theorem 1.3. Thus, assume that $S$ is biquadrangle free.

We can use (3.3) and (3.5) to list all combinatorial types of pencils $\Gamma$ of degree 4; then, Propositions 3.7 and 2.8 imply the following lemma.

Lemma 7.7 The cardinality $|\Gamma|$ is bounded as follows:

$$
\begin{array}{rcccc}
D \in & \{6 \bmod 4\} & \{4 \bmod 4\} & \{7 \bmod 4\} & \{5 \bmod 4\} \\
\max |\Gamma|= & 24 & 22 & 20 & 18
\end{array}
$$

If $\Gamma$ has at least one simple section, the values of $D$ are bounded as follows:

- if $|\Gamma| \geqslant 22$, then $D=4$;

- if $|\Gamma|=21$, then $D \in\{2,4,6\}$;

- if $|\Gamma|=19,20$, then $D \in\{2,4,6,8\}$;

- if $|\Gamma|=17,18$, then $D \in\{2$.. 10\}.

Lemmas 7.5 and 7.7 imply the statement of Theorem 7.1 for $D \geqslant 9$.

For the remaining few values of $D$, including $D=2$, we run the algorithm of Sect. A.2, taking (7.2) for the defining property $\mathfrak{P}$. The threshold is $|\mathrm{Fn} S|>\bar{M}$, where $\bar{M}:=\bar{M}(D)$ is the bound stated in the theorem; then, in view of Lemma 7.5, we can assume that $|\Gamma|>\bar{M}_{\Gamma}:=16$. (For $D=6$ or 8 we can increase $\bar{M}_{\Gamma}$ to 20 or 18 , respectively.) Furthermore, assuming (7.1) and taking $l_{1}$ for $l$, we have

$$
|\sec l|=\left|\sec _{1} l\right| \geqslant v_{\min }:=\left\lceil\frac{1}{4}(\bar{M}+1-|\Gamma|)\right\rceil .
$$

Configurations of low rank (Step 3) are analyzed as described in Sect. A.4.2. The result is the conclusion that $\mid$ Fn $S \mid \leqslant \bar{M}(D)$ unless $D=4$ and Fn $S \cong \Theta_{36}^{\prime}, \Theta_{36}^{\prime \prime}, \Theta_{34}^{\prime}, \Theta_{33}$, $\Theta_{32}$, as stated. As a by-product (cf. Sect. A.1), we obtain Addendum 7.2. 


\subsection{Spatial Quartis}

If $X \subset \mathbb{P}^{3}$ is a smooth quartic and Fn $X$ is triangle free, the best known bound is $\mid$ Fn $X \mid \leqslant 52$, whereas the best example so far had 33 lines. (Example 7.9 has 37 lines.) The last proof applies to $D=2$, and we state this fact separately (combining it with the results of the previous sections). This leaves configurations with a biquadrangle as the only case still open.

Proposition 7.8 Let $X \subset \mathbb{P}^{3}$ be a smooth quartic, and assume that Fn $X$ has no triangles or biquadrangles. Then $|\mathrm{Fn} X| \leqslant 36$, and this bound is sharp.

Example 7.9 There exists a smooth spatial quartic $X \subset \mathbb{P}^{3}$ with the graph Fn $X$ triangle free and $|\mathrm{Fn} X|=37$. This configuration, found in [4], was obtained during the experiments leading to the proof of Lemma 7.6.

\section{Triangular Configurations}

Recall that a graph $\Gamma$ and the corresponding configuration are called triangular if $\operatorname{girth}(\Gamma)=3$. Large triangular configurations of lines in smooth quartics have been studied in [5] (see $D=2$ in Table 1). Therefore, we assume that $D \geqslant 3$.

\subsection{Statements}

The following theorem is the principal result of this section.

Theorem 8.1 (see Sect. 8.3) Let $X \subset \mathbb{P}^{D+1}$ be a smooth 2D-polarized K3-surface, $D \geqslant 3$, and assume that the Fano graph Fn $X$ is triangular, i.e., girth $(\operatorname{Fn} X)=3$. Then, with few exceptions, we have the following sharp bounds:

$$
\begin{array}{rlccccc}
D & = & 3 & 4 & 5 & \{7 \bmod 3\} & \text { else } \\
\mid \text { Fn } X \mid & \leqslant & 36 & 29 & 24 & 24 & 21
\end{array}
$$

The exceptions are $D=3$, Fn $X \cong \Psi_{42}, \Psi_{38}$ and $D=4$, Fn $X \cong \Psi_{33}$ (see Table 1 ).

Addendum 8.2 (see Sect. 8.3) If $D=3$, the number of lines in triangular configurations takes all values in $\{3 . .36,38,42\}$.

The graph $\Psi_{42}$ is 9-regular; it contains two minimal pencils, which are both of type $3 \widetilde{\mathbf{A}}_{2}+3 \mathbf{A}_{1}$. The corresponding equilinear family contains models of the two discriminant minimizing surfaces $X([2,1,20])$ and $X([6,3,8])$ (see [3]).

\subsection{Lines Intersecting a Triangle}

Consider a geometric configuration $S$ and a triangle $\Sigma=\left\{l_{1}, l_{2}, l_{3}\right\} \subset$ Fn $S$. Let $\Gamma:=\mathcal{P}(\Sigma)$. The next lemma is an application of Proposition 2.8 to simple test configurations (cf. also Proposition 2.11). 
Table 6 Pencils of degree 3 with a section

\begin{tabular}{lll}
\hline$|\Gamma|$ & $\Gamma$ & $D$, remarks \\
\hline 24 & $8 \widetilde{\mathbf{A}}_{2}$ & $4, s \in \mathcal{F}_{h}(\Gamma) \otimes \mathbb{Q}$ \\
22 & $7 \widetilde{\mathbf{A}}_{2}+\mathbf{A}_{1}$ & $4, s \in \mathcal{F}_{h}(\Gamma) \otimes \mathbb{Q}$ \\
21 & $7 \widetilde{\mathbf{A}}_{2}$ & $5, s \in \mathcal{F}_{h}(\Gamma) \otimes \mathbb{Q}$ \\
& $6 \widetilde{\mathbf{A}}_{2}+3 \mathbf{A}_{1}$ & 3,4 \\
20 & $6 \widetilde{\mathbf{A}}_{2}+2 \mathbf{A}_{1}$ & $2,3,4$ \\
19 & $6 \widetilde{\mathbf{A}}_{2}+\mathbf{A}_{1}$ & $2,3,4,5$ \\
& $5 \widetilde{\mathbf{A}}_{2}+4 \mathbf{A}_{1}$ & 3,4 \\
\hline
\end{tabular}

Lemma 8.3 Assume that $D \geqslant 3$. Then any section of $\Gamma$ is simple. Furthermore,

(1) each subgraph $\sec l_{i}, 1 \leqslant i \leqslant 3$, is discrete;

(2) $\left(\sec l_{i}\right) *\left(\sec l_{j}\right) \leqslant 1$ for $1 \leqslant i \neq j \leqslant 3$;

(3) if $D \geqslant 4$, then $\sec \Gamma$ is discrete.

Lemma 8.4 We have the following sharp bounds on the cardinality $|\sec \Gamma|$ :

$$
\begin{array}{rlcccccc}
D & = & 3 & 4 & 5,6 & 7 . .11 & \text { else } \\
|\sec \Gamma| & \leqslant & 21 & 9 & 3 & 1 & 0
\end{array}
$$

If $D=3$ and $|\sec \Gamma| \geqslant 20$, then either $\mathrm{Fn} S \cong \Psi_{42}$ or $|\mathrm{Fn} S| \leqslant 36$.

Proof If $D \geqslant 4$, then, in view of Lemma 8.3(3), all statements easily follow from Proposition 2.8. If $D=3$, then Lemma 8.3(1), (2) means that $\Sigma$ is suitable for enumeration (see Sect. A.5), and we use the algorithm of Sect. A.5.

\subsection{Proof of Theorem 8.1 and Addendum 8.2}

Using Proposition 2.8 and bounds (3.3), (3.5), we can compile a list of large pencils $\Gamma:=\mathcal{P}(\Sigma)$ admitting a section; the result is shown in Table 6. (In the first three lines, we indicate that any section lies in $\mathcal{F}_{h}(\Gamma) \otimes \mathbb{Q}$ and, hence, $\mathcal{F}_{h}(\Gamma)$ has no geometric extensions of positive corank.) Comparing this with Corollary 3.8 and Lemma 8.4, we conclude that, for $D \geqslant 6$, the maximal cardinality $|\mathrm{Fn} X|$ is realized by a parabolic configuration and is as stated in the theorem. Similarly, if $D=5$, the maximum $\mid$ Fn $X \mid=24$ is given by the only geometric finite index extension of $7 \widetilde{\mathbf{A}}_{2}$, which has three sections.

The case $D=4$ is considered in Sect. 5.4, resulting in Theorem 1.3. Thus, we assume that $D=3$ and $\mid$ Fn $X \mid>\bar{M}:=36$. In view of Lemma 8.4, we can also assume that $|\sec \Gamma| \leqslant \bar{M}_{\mathrm{sec}}:=19$ and, hence, $|\Gamma|>\bar{M}_{\Gamma}:=17$. Indexing the lines so that $\left|\sec l_{1}\right| \geqslant\left|\sec l_{2}\right| \geqslant\left|\sec l_{3}\right|$, we apply the algorithm of Sect. A.2, taking for $l$ the line $l_{1}$ and estimating the number of sections via

$$
|\sec l| \geqslant v_{\min }:=\left\lceil\frac{1}{3}(37-|\Gamma|)\right\rceil .
$$

The final step of the algorithm (see Sect. A.2.3) is explained in Sect. A.4.1. 


\subsection{Proof of Theorem 1.1 and Addendum 1.2}

The case of spatial quartics is treated in [5]. For the other degrees, Theorem 1.1 is proved by comparing the bounds given by Theorems 4.4-4.6 (locally elliptic graphs), 4.5 (astral graphs), and 6.1, 7.1, and 8.1 (pentagonal, quadrangular, and triangular graphs, respectively).

Addendum 1.2 is given by [5] (for $D=2$ ) or Addendum 7.2 (for $D=3,4$ ).

\subsection{Proof of Theorem 1.4}

We use Theorem 2.7 and detect the extremal graphs in Table 1 realized by configurations of real lines in real surfaces (marked with a $\$$ in the table). If such a graph does not exist, we merely state that $M_{\mathbb{R}}(D)=\bar{M}(D)$ is the auxiliary bound in Theorem 1.1. The sharpness of this bound for $D=8$ and $D>10$ is given by Addendum 4.7.

If $D=5$, the Fano graph $\Phi_{28}$ of the discriminant minimizing surface $X([2,0,16])$ (see Theorem 1.7 and [4]) is real. If $D=7$, a certain real configuration $\Phi_{26}$ (see [4]) is found in the course of the proof of Theorem 6.1 .

\section{Appendix A: Algorithms}

This appendix outlines a few technical details concerning the algorithms used to enumerate large pencils or sets of sections. All computations were done in GAP [7].

\section{A.1 Defining Properties}

In the enumeration routines, we are interested in the configurations satisfying a certain hereditary defining property $\mathfrak{P}$ (such as locally elliptic, triangle free, quadrangle free, etc.) We incorporate this property as part of Algorithm 2.5: upon establishing the admissibility of a configuration $S$, we compute the graph Fn $S$ and disregard $S$ as invalid if $\mathfrak{P}(S)$ does not hold.

Since Algorithm 2.6 relies upon Algorithm 2.5, we will essentially speak about $\mathfrak{P}$ (sub-) geometric configurations, i.e., those admitting a geometric finite index extension satisfying the defining property $\mathfrak{P}$.

For statistical purposes (e.g., for establishing the sharpness of the bounds), in Algorithm 2.6 we keep track of the sizes of all geometric configurations: as soon as a $\mathfrak{P}$-geometric configuration $S$ is discovered, its cardinality $|\mathrm{Fn} S|$ is recorded.

\section{A.2 Pencils}

As an essential part of most proofs, we enumerate all $\mathfrak{P}$-geometric configurations $S$ spanned by a pencil $\Gamma:=\mathcal{P}(\Sigma)$ with a distinguished fiber $\Sigma$ and a number of sections $s_{i} \in \sec _{1} \Gamma$ such that

$$
|\Gamma|>\bar{M}_{\Gamma}, \quad \mid \text { Fn } S \mid>\bar{M},
$$


where $\bar{M}_{\Gamma}$ and $\bar{M}$ are certain thresholds fixed in advance. The types of the fibers of $\Gamma$ are given by Corollaries 3.3 and 3.4, and the possible combinatorial types of pencils are listed using (3.3)-(3.5) (see also Remark 3.5).

Thus, we fix a combinatorial type $\Gamma \supset \Sigma$ and change the defining property $\mathfrak{P}$ to

$$
\mathfrak{P}_{\Gamma}: S \longmapsto \mathfrak{P}(S) \text { and } \Gamma \subset \text { Fn } S \text { is a maximal parabolic subgraph. }
$$

We also fix a line $l \in \Sigma$ and, assuming that $\left|\sec _{1} l\right| \geqslant\left|\sec _{1} l^{\prime}\right|, l^{\prime} \in \Sigma$, in the resulting configuration $S$, determine a lower bound $v_{\min } \leqslant\left|\sec _{1} l\right|$ on the number of simple sections through $l$ that is necessary for the inequality $|\mathrm{Fn} S|>\bar{M}$ in (A.1).

\section{A.2.1 Step 1}

We start with the lattice $S_{0}$ and stabilizer $G_{0}$ :

$$
S_{0}:=\mathcal{F}_{h}(\Gamma), \quad G_{0}:=\operatorname{stab}\{l\} \subset \text { Aut } \Gamma
$$

Then, sections $s_{1}, s_{2}, \ldots$ intersecting $l$ are added to $\Gamma$ one by one, and we consider consecutive overlattices and subgroups

$$
S_{i}:=\left(S_{i-1}+\mathbb{Z} s_{i}\right) / \operatorname{ker} \supset S_{i-1}, \quad G_{i}:=\operatorname{stab}\left\{s_{1}, \ldots, s_{i}\right\} \subset G_{0} .
$$

At each step, we require that

$$
S_{i} \text { should be } \mathfrak{P}_{\Gamma} \text {-subgeometric (cf. Sect. A.1) and rk } S_{i}>\operatorname{rk} S_{i-1} \text {. }
$$

This process continues until no other section satisfying (A.2) can be added.

In all cases, the set $\sec l$ is discrete (cf. Sect. A.5(1)) and, hence, a section $s$ is described by its coordinates $\bar{s} \subset \Gamma$ (see Convention 3.6), so that $l \in \bar{s}$. At each step, we try for $s_{i}$ a single representative of each $G_{i-1}$-orbit. We also check partially the defining property $\mathfrak{P}$, leaving the ultimate validation to Algorithm 2.6, where the group $G_{i}$ is used to reduce the number of candidates to be analyzed.

Remark A.1 As a technical tool reducing the computation and overcounting, we fix an integer $2 \leqslant p \leqslant 4$ (depending on the size $\left|G_{0}\right|$ ) and store, during the $i$-th step, the $G_{0^{-}}$ orbits of all $i$-tuples $\left\{s_{1}, \ldots, s_{i}\right\}, i \leqslant p$, of sections satisfying (A.2). These pools $P_{i}$ are used two-fold: first, during the $i$-th step (if $i \leqslant p$ ), we disregard the orbits that have already been stored; second, when collecting the candidates $s_{i+1}$ to extend a set $\left\{s_{1}, \ldots, s_{i}\right\}$, we select only those $s_{i+1} \in P_{1}$ for which each subset $s \subset\left\{s_{i}, \ldots, s_{i+1}\right\}$ of cardinality $n \leqslant \min (i, p)$ is in the pool $P_{n}$.

\section{A.2.2 Step 2}

We use Algorithm 2.6 to compute all $\mathfrak{P}_{\Gamma}$-geometric finite index extensions of all configurations $S$ obtained at all stages of Step 1. Most configurations violating the hypothesis $|\mathrm{Fn} S| \leqslant \bar{M}$ are found at this point. Then, disregarding the configurations 
of rank 20 (as admitting no further extensions) and those in which $|\sec l|<v_{\min }$ (as not meeting the goal), we replace each remaining geometric extension with the respective lattice $\mathcal{F}_{h}(\Gamma \cup \sec l)$. The sets $\sec l$ are sorted again, retaining a single representative of each $G_{0}$-orbit.

\section{A.2.3 Step 3}

The remaining configurations have rank 19 or 18 . We pick another line $l^{\prime} \in \Sigma$ and try to add up to two independent extra sections $e_{i} \in \sec l^{\prime}$. (The choice of $l^{\prime}$ is explained and justified in Sect. A.4.) Thus, we start with

$$
S_{0}:=\mathcal{F}_{h}\left(\Gamma \cup\left\{s_{1}, \ldots, s_{n}\right\}\right), \quad G_{0}:=\operatorname{stab}\left\{s_{1}, \ldots, s_{n}\right\} \subset \operatorname{stab}\left(l, l^{\prime}\right) \times \mathbb{S}(\sec l)
$$

and add one or two extra sections as in Step 1, obtaining consecutive lattices $S_{i}$ and groups $G_{i}$. In addition to (A.2), we require that the set $\sec _{1} l$ should remain constant (as otherwise $S_{i}$ is among the configurations considered at Steps 1 and 2). Finally, an analogue of Step 2 is applied to all new lattices obtained.

Remark A.2 The extra sections $e_{i}$ may intersect the original sections $s_{k}$; hence, an extra section $e$ is determined by its coordinates $\bar{e} \subset \Gamma$ (cf. Convention 3.6) and a subset of $\left\{s_{1}, \ldots, s_{n}\right\}$. The cardinality of the latter is usually bounded by the defining property $\mathfrak{P}$ (cf. Sect. A.5(2)).

\section{A.3 Pencils with Disjoint Sections}

If all sections of interest are known to be pairwise disjoint (e.g., in the treatment of the locally elliptic configurations, see Sect. 4.4), the algorithm of Sect. A.2 can be modified. Namely, we start with a pencil $\Gamma$ and a distinguished fiber $\Sigma=\left\{l_{1}, \ldots, l_{n}\right\}$ and fix the goals $v_{i} \leqslant\left|\sec l_{i}\right|, i=1, \ldots, n$. Then, disjoint sections are added one by one as in Sect. A.2.1, except that we do not require that the rank of the configuration should increase at each step. As soon as a configuration $S$ of the maximal rank 20 is obtained, it is analyzed as in Sect. A.2.2. Configurations of lower rank that do not meet the goals are discarded as small.

\section{A.4 Triangular and Quadrangular Configurations}

A posteriori, it turns out that it is only triangular (for $D=3$ ) and quadrangular configurations that need the algorithm of Sect. A.2 to full extent. Below, we explain and justify the choice of the additional line(s) $l^{\prime}, l^{\prime \prime} \in \Sigma$ for Step 3 (see Sect. A.2.3).

\section{A.4.1 Triangular Configurations (See Sect. 8.3)}

Let $\Sigma:=\left(l_{1}, l_{2}, l_{3}\right)$ be the triangle used in the proof, so that $l=l_{1}$. Left to Step 3 are four configurations $S_{0}$ of rank 19 and three configurations of rank 18. In the former case, a single extra section is to be added and, by the obvious symmetry, we can use $l^{\prime}=l_{2}$. 
If $\mathrm{rk} S_{0}=18$, then, in all three cases, $\sec l_{2}=\sec l_{3}=\varnothing$ in each geometric finite index extension $S_{0}^{\prime} \supset S_{0}$. In two cases, $\sec l_{2} \neq \varnothing$ and $\sec l_{3} \neq \varnothing$ in each geometric corank 1 extension $S_{1}^{\prime} \supset S_{0}$; hence, any geometric corank 2 extension is generated over $S_{0}$ and $\mathbb{Q}$ by two sections adjacent to the same line, which, by symmetry, can be chosen to be $l_{2}$. In the exceptional case, we have $\Gamma \cong 5 \widetilde{\mathbf{A}}_{2}+3 \mathbf{A}_{1}$ and

$$
\left|\sec l_{1}\right|=7, \quad\left|\sec l_{2}\right|=1, \quad\left|\sec l_{3}\right|=0 ;
$$

hence, if a geometric corank 2 extension $S_{2}^{\prime} \supset S_{0}$ is not generated by two sections in the same set, we have $|\sec \Gamma| \leqslant 9$ and $\mid$ Fn $S \mid \leqslant 27$.

\section{A.4.2 Quadrangular Configurations (See Sects. 7.3 and 7.4)}

Let $\Sigma:=\left(l_{1}, l_{2}, l_{3}, l_{4}\right)$ be the quadrangle used in the proofs, with the numbering satisfying (7.1) except that, for Lemma 7.6, we lift the restriction val $l_{1} \geqslant \operatorname{val} l_{2}$. Each configuration $S_{0}$ to be considered at Step 3 has $\mathrm{rk} S_{0} \geqslant 18$, but the number of configurations is quite large. In order to avoid tedious case-by-case analysis, we merely add up to two extra sections $e^{\prime} \in \sec l^{\prime}, e^{\prime \prime} \in \sec l^{\prime \prime}$ trying all pairs $\left(l^{\prime}, l^{\prime \prime}\right)$; since val $l_{2} \geqslant$ val $l_{4}$ by (7.1), it suffices to consider

$$
\left(l^{\prime}, l^{\prime \prime}\right)=\left(l_{2}, l_{2}\right), \quad\left(l_{2}, l_{3}\right), \quad\left(l_{2}, l_{4}\right), \quad\left(l_{3}, l_{3}\right)
$$

(If rk $S_{0}=19$, i.e., only one extra section is to be added, we try $l^{\prime}=l_{2}$ or $l_{3}$.) The maximal number of original sections $s_{i} \in \sec l_{1}$ that an extra section $s^{*} \in \sec l^{*}$ may intersect (see Remark A.2) is given by Lemma 7.4(3)-(5), (7), depending on the degree $h^{2}=2 D$, line $l^{*}=l_{2}$ or $l_{3}$, and whether the resulting configuration is or is not allowed to have biquadrangles. If $l \neq l^{\prime \prime}$, we also need to take into account the intersection $e^{\prime} \cdot e^{\prime \prime}=0$ or 1 . These complications slow the computation down, but it still remains reasonably feasible.

\section{A.5 Sets of Sections}

Another part of the proofs is the classification of sets of sections in the presence of a fixed parabolic subgraph (affine Dynkin diagram) $\Sigma$ and a hereditary defining property $\mathfrak{P}$.

Fix an ordering $\Sigma=\left\{l_{1}, \ldots, l_{n}\right\}$ of the lines constituting $\Sigma$. We say that $\Sigma$ is suitable for enumeration if its sections in any $\mathfrak{P}$-geometric configuration have the following properties:

(1) each subgraph $\sec l_{i}, 1 \leqslant i \leqslant n$, is discrete, and

(2) $\left(\sec l_{i}\right) *\left(\sec l_{j}\right) \leqslant 1$ for $1 \leqslant i \neq j \leqslant n$.

\section{A.5.1 Steps $1_{1}, 1_{2}$}

Assuming these properties, we fix the goals $v_{i}=\left|\sec l_{i}\right|$ and start with the lattice

$$
S_{2}:=S_{2,0}:=\mathcal{F}_{h}\left(\Sigma \cup \sec l_{1} \cup \sec l_{2}\right),
$$


where $\sec l_{i}=\left\{s_{i 1}, \ldots, s_{i} v_{i}\right\}$ consists of $v_{i}$ pairwise disjoint sections, $i=1,2$, and $s_{1 k} \cdot s_{2 k}=1$ for $1 \leqslant k \leqslant r$ (another parameter fixed in advance) and $s_{1 p} \cdot s_{2 q}=0$ for all other pairs $(p, q)$. Let $G_{2}=\mathbb{S}_{r} \times \mathbb{S}_{v_{1}-r} \times \mathbb{S}_{v_{2}-r}$ be the group of symmetries of this configuration.

\section{A.5.2 Step $1_{m}, 3 \leqslant m \leqslant n$}

At this step, the previously constructed configuration $S_{m-1}=S_{m-1,0}$ is considered frozen, i.e., we change the defining property $\mathfrak{P}$ to

$$
\mathfrak{P}_{m-1}: S \longmapsto \mathfrak{P}(S) \text { and }\left|\sec l_{k}\right|=v_{k} \text { for } k<m \text {. }
$$

We add to $S_{m-1}$, one by one, exactly $v_{m}$ pairwise disjoint sections $s_{m i} \in \sec l_{m}$ and consider consecutive overlattices $S_{m-1, i}:=\left(S_{m-1, i-1}+\mathbb{Z} s_{m, i}\right) /$ ker. Unlike (A.2), we do not require that the ranks should increase. Each section $s_{m i}$ is determined by an $(m-1)$-tuple of at most one-element sets $\left[s_{m i}\right]_{k} \subset \sec l_{k}, k<m$. Since the symmetry groups involved are typically much smaller than those in Sect. A.2, we use a more aggressive algorithm: at each step, we collect the candidates $s_{m i}$ extending all previously constructed sets $\left\{s_{m 1}, \ldots, s_{m, i-1}\right\}$, compute $G_{m-1}$-orbits of all $i$-tuples $\left\{s_{m 1}, \ldots, s_{m i}\right\}$ obtained, and choose one representative of each orbit. We also use pools $P_{i}, i \leqslant 4$, as in Remark A.1.

Upon completion of this step, we obtain a collection of lattices $S_{m}:=S_{m-1, v_{m}}$ and stabilizers $G_{m}$,

$$
S_{m}:=\left(S_{m-1}+\mathbb{Z} \sec l_{m}\right) / \text { ker, } \quad G_{m}:=\operatorname{stab} \sec l_{m} \subset G_{m-1},
$$

to be used at Step $1_{m+1}$. (We retain only those configurations $S_{m}$ which admit a $\mathfrak{P}$ geometric extension with exactly $v_{k}$ sections intersecting $l_{k}, k \leqslant m$.)

If only sets of sections are to be classified (e.g., in order to determine their maximal number), we stop at this point. Otherwise, if we are interested in all $\mathfrak{P}_{n}$-geometric extensions $S$ of the lattices $S_{n}$ obtained satisfying an inequality

$$
\mid \text { Fn } S \mid>\bar{M}
$$

fixed in advance, we proceed similar to Sect. A.2.

\section{A.5.3 Step 2}

For each lattice $S_{n}$ obtained at the final step $1_{n}$, compute all $\mathfrak{P}_{n}$-geometric finite index extensions (by Algorithm 2.6); then, select those satisfying (A.3). After this step, we disregard all lattices of rank 20.

\section{A.5.4 Step 3}

For the remaining configurations $S_{n}:=S_{n, 0}$, we increase the rank by adding, one by one, $\left(20-\mathrm{rk} S_{n}\right)$ disjoint extra lines (i.e., lines $e_{i}$ disjoint from all $\left.l_{1}, \ldots, l_{n}\right)$ and 
considering the configurations

$$
S_{n, i}:=\left(S_{n, i-1}+\mathbb{Z} e_{i}\right) / \operatorname{ker}
$$

and all their $\mathfrak{P}_{n}$-geometric finite index extensions computed by Algorithm 2.6. At each step, we require that $\mathrm{rk} S_{n, i}>\mathrm{rk} S_{n, i-1}$. An extra line $e$ can be regarded as a subset $[e] \subset \sec \Sigma$ (cf. Convention 3.6; the cardinality of this subset and/or its intersections with $\sec l_{i}$ is usually bounded by geometric arguments), and we use an aggressive algorithm similar to Sect. A.5.2.

In the rest of this section, we justify the fact that, if rk $S_{n}=18$, it suffices to add pairs of disjoint extra lines $e_{1}, e_{2}$, keeping the computation feasible. The other cases, where rk $S_{n} \leqslant 17$, are considered below when they appear.

We say that a $\mathfrak{P}_{n}$-geometric corank $r$ extension $S^{\prime} \supset S_{n}$ is well generated if it has a good basis, i.e., a collection of pairwise disjoint lines $e_{1}, \ldots, e_{r} \in \mathcal{P}_{S^{\prime}}(\Sigma) \backslash \Sigma$ generating $S^{\prime}$ over $S_{n}$ and $\mathbb{Q}$. Any line appearing in a good basis is called generating. Given a good basis $\left\{e_{1}, \ldots, e_{r}\right\}$, we denote by $E_{i}:=E\left(e_{i}\right)$ the connected component of the pencil $\mathcal{P}_{S^{\prime}}(\Sigma)$ containing the vertex $e_{i}, i=1, \ldots, r$. If $r=1$, we consider also the union $E_{\mathrm{gen}}\left(S^{\prime}\right)=\bigcup_{e} E(e)$ over all generating lines $e$.

Let $\mathcal{T}(\Sigma)$ be the set of all types of fibers that can occur in the pencil $\mathcal{P}(\Sigma)$ (in any $\mathfrak{P}_{n}$-geometric configuration), see Corollaries 3.3 and 3.4.

Lemma A.3 Given $S_{n}$ as above and a $\mathfrak{P}_{n}$-geometric corank 1 extension $S^{\prime} \supset S_{n}$, assume that, for any inclusion $E_{\mathrm{gen}}\left(S^{\prime}\right) \hookrightarrow \Sigma^{\prime}$ to a graph $\Sigma^{\prime} \in \mathcal{T}(\Sigma)$, we have

$$
\left|\Sigma^{\prime}\right|-\left|E_{\text {gen }}\left(S^{\prime}\right)\right| \leqslant \bar{M}-\mid \text { Fn } S^{\prime} \mid
$$

Then, any $\mathfrak{P}_{n}$-geometric corank 2 extension $S^{\prime \prime} \supset S^{\prime} \supset S_{n}$ satisfying (A.3) is well generated over $S_{n}$.

Proof An extension $S^{\prime \prime} \supset S^{\prime}$ is well generated over $S_{n}$ if and only if there is a line $e_{2} \in \mathcal{P}_{S^{\prime \prime}}(\Sigma) \backslash \mathcal{P}_{S^{\prime}}(\Sigma)$ disjoint from at least one generating line $e_{1}$ of $S^{\prime}$. If this is not so, all new lines $e_{2}$ and all generating lines $e_{1}$ must be in the same fiber $\Sigma^{\prime}$ of $\mathcal{P}_{S^{\prime \prime}}(\Sigma)$, and we have $\mid$ Fn $S^{\prime \prime}|\leqslant|$ Fn $S^{\prime}|+| \Sigma^{\prime}|-| E_{\text {gen }}\left(S^{\prime}\right) \mid \leqslant \bar{M}$.

Corollary A.4 Under the assumptions of Lemma A.3, if $E_{\mathrm{gen}}\left(S^{\prime}\right)$ does not admit an embedding into $\Sigma^{\prime} \in \mathcal{T}(\Sigma)$, then any $\mathfrak{P}_{n}$-geometric extension $S^{\prime \prime} \supset S^{\prime} \supset S_{n}$ of corank 2 is well generated over $S_{n}$.

\section{A.6 Quadrangular Configurations}

In the proof of Lemma 7.5, for $D=4$ we need to use Step 3 of the previous algorithm to list all geometric configurations $S$ containing a pencil $\Gamma:=\mathcal{P}(\Sigma), \Sigma \cong \widetilde{\mathbf{A}}_{3}$, and such that $\mid$ Fn $S \mid>30$ and $|\sec \Gamma| \geqslant 15$. In this case, the rank rk $S_{4}$ can be as low as 15 , so that we may need to add up to five extra lines, and Lemma A.3 does not always apply.

Fix a configuration $S_{4}$ obtained at Step $1_{4}$. Recall that we consider triangle free configurations and insist that the set of sections of the pencil does not increase; in 
other words, we take for the defining property (depending on $S_{4}$ or, more precisely, on the fixed goals $v_{i}=\left|\sec l_{i}\right|$ in $\left.S_{4}, 1 \leqslant i \leqslant 4\right)$

$$
\mathfrak{P}_{4}: S \longmapsto S \text { is triangle free and } \sec l_{i}=v_{i} \text { in } S, 1 \leqslant i \leqslant 4 \text {. }
$$

In the notation introduced prior to Lemma A.3, we have $E_{i} \cong \widetilde{\mathbf{A}}_{3}, \mathbf{A}_{2}$, or $\mathbf{A}_{1}$ for any good basis $\left\{e_{1}, \ldots e_{n}\right\}$, and the following two statements are proved by a simple analysis of the modifications of the components under $\mathfrak{P}_{4}$-geometric extensions. (The hypotheses of both statements are to be checked for geometric extensions, i.e., those obtained after applying Algorithm 2.6.)

Lemma A.5 Assume that a $\mathfrak{P}_{4}$-geometric corank $r$ extension $S^{\prime} \supset S_{4}$ has a good basis $e_{1}, \ldots, e_{r}$, and let $\bar{r}:=\#\left\{i \leqslant r \mid E_{i} \cong \mathbf{A}_{1}\right\}$. Then any $\mathfrak{P}_{4}$-geometric corank 1 extension $S^{\prime \prime} \supset S^{\prime}$ such that $\left|\mathrm{Fn} S^{\prime \prime}\right|>\left|\mathrm{Fn} S^{\prime}\right|+2 \bar{r}$ is well generated over $S_{4}$.

Proof In the worst case scenario, a component $E_{i} \cong \mathbf{A}_{1}$ extends to $E_{i}^{\prime \prime} \cong \widetilde{\mathbf{A}}_{3}$, so that the line $e_{i}^{\prime \prime} \in E^{\prime \prime}$ opposite to $e_{i}$ is already in $\mathcal{P}_{S^{\prime}}(\Sigma)$, and all new components are of this form. In this case, $\left|\mathrm{Fn} S^{\prime \prime}\right| \leqslant\left|\mathrm{Fn} S^{\prime}\right|+2 \bar{r}$.

Corollary A.6 Assume that a $\mathfrak{P}_{4}$-geometric corank $r$ extension $S^{\prime} \supset S_{4}$ has a good basis such that $\left|E_{i}\right| \geqslant 2,1 \leqslant i \leqslant r$. Then any $\mathfrak{P}_{4}$-geometric corank 1 extension $S^{\prime \prime} \supset S^{\prime}$ is well generated over $S_{4}$.

End of the proof of Lemma 7.5 We start from a configuration $S_{4}$, rk $S_{4} \geqslant 15$, and add extra lines one by one, verifying, at each step, that all subsequent corank 1 extensions are still well generated.

If rk $S_{4}=19$, there is nothing to prove: all extensions are well generated.

If rk $S_{4}=18$, then every $\mathfrak{P}_{4}$-geometric corank 1 extension $S^{\prime} \supset S_{4}$ either satisfies the hypotheses of Corollary A.4 or A.6 or has $\left|\mathrm{Fn} S^{\prime}\right| \leqslant 28$, and then we can use Lemma A.5; thus, any $\mathfrak{P}_{4}$-geometric corank 2 extension $S^{\prime \prime} \supset S_{4}$ that satisfies the inequality $\left|\mathrm{Fn} S^{\prime \prime}\right|>30$ is well generated.

If rk $S_{4}=15$ or 16 , then, step-by-step, one shows that, for each $\mathfrak{P}_{4}$-geometric extension $S^{\prime \prime \prime} \supset S_{4}$, one has $|E| \geqslant 2$ for each connected component $E \subset \mathcal{P}_{S^{\prime \prime \prime}}(\Sigma)$; then, by Corollary A.6, all extensions at the next step are also well generated.

Finally, let rk $S_{4}=17$ and pick a $\mathfrak{P}_{4}$-geometric corank 1 extension $S^{\prime} \supset S_{4}$. The computation shows that, if $S^{\prime}$ does not satisfy the hypotheses of Lemma A.3 or Corollary A.6, then $E_{\text {gen }}\left(S^{\prime}\right) \cong \mathbf{A}_{1}$ or $2 \mathbf{A}_{1}$ and $\left|\mathrm{Fn} S^{\prime}\right| \leqslant 26$. By Lemma A.5, any extension $S^{\prime \prime \prime} \supset S^{\prime}$ such that $\left|\mathrm{Fn} S^{\prime \prime \prime}\right|>30$ contains a well-generated $\mathfrak{P}_{4}$-geometric corank 2 extension $S^{\prime \prime} \supset S^{\prime} \supset S$ (i.e., there is a line $l \in \mathcal{P}_{S^{\prime \prime}}(\Sigma) \backslash \mathcal{P}_{S^{\prime}}(\Sigma)$ disjoint from at least one good generator of $S^{\prime}$ ).

Thus, let $S^{\prime \prime} \supset S_{4}$ be a well-generated $\mathfrak{P}_{4}$-geometric corank 2 extension. In all but a few cases, Lemma A.5 implies that any further extension $S^{\prime \prime \prime} \supset S^{\prime \prime}$ is well generated whenever $\left|\mathrm{Fn} S^{\prime \prime \prime}\right|>30$. In each exceptional case, the graph $G \subset \Gamma_{S^{\prime \prime}}(\Sigma)$ of generating lines splits, $G=G_{1} \cup G_{2}, G_{1} \cap G_{2}=\varnothing$, so that

$$
G_{1}=\left\{e_{1}^{\prime}, e_{1}^{\prime \prime}\right\} \cong 2 \mathbf{A}_{1}, \quad G_{2} \cong 2 \mathbf{A}_{1}, \widetilde{\mathbf{A}}_{3}+\mathbf{A}_{2}, \text { or } 2 \widetilde{\mathbf{A}}_{3}
$$


and good are the bases of the form $\left\{e_{1}, e_{2}\right\}$, where $e_{1} \in G_{1}, e_{2} \in G_{2}$. (If $G_{2} \cong$ $2 \mathbf{A}_{1}$, then we also have $\left|\mathrm{Fn} S^{\prime \prime}\right|=28$.) A simple argument shows that any offending extension $S^{\prime \prime \prime} \supset S^{\prime \prime}$ is generated by an extra line $e_{2}$ such that $e_{2} \cdot e_{1}^{\prime}=e_{2} \cdot e_{1}^{\prime \prime}=1$, so that $e_{1}^{\prime}, e_{2}, e_{1}^{\prime \prime}$ are part of a quadrangle. This observation limits the number of choices for $e_{2}$; we do list all such extensions (with the intersections of the extra lines prescribed) and find out that they are never $\mathfrak{P}_{4}$-geometric.

\section{A.7 Pentagonal Configurations}

In the proof of Lemma 6.4, where the fiber $\Sigma \cong \widetilde{\mathbf{A}}_{4}$ is a pentagon, we encounter configurations $S_{5}$ of rank rk $S_{5} \geqslant 17$. The following statement is a refinement of Lemma A.3 using the known structure of $\Sigma$. We assume that the defining property is $\mathfrak{P}: S \mapsto\left(\right.$ girth $($ Fn $S)=5$ ) and consider a subgeometric configuration $S_{5}$ obtained after Step $1_{5}$ of the algorithm of Sect. A.5. The other notations and terminology are introduced prior to Lemma A.3.

Lemma A.7 Let $S_{5}$ be as above and $S^{\prime} \supset S_{5}$ a $\mathfrak{P}_{5}$-geometric corank 1 extension. Define $\delta\left(S^{\prime}\right)$ as follows:

- $\delta\left(S^{\prime}\right)=2$ if $E_{\text {gen }} \cong \mathbf{A}_{1}$;

- $\delta\left(S^{\prime}\right)=1$ if $E_{\text {gen }} \cong 2 \mathbf{A}_{1}$ or $E_{\text {gen }} \cong \mathbf{A}_{2}$ with only one line generating;

- $\delta\left(S^{\prime}\right)=0$ in all other cases.

Then, any $\mathfrak{P}_{5}$-geometric corank 2 extension $S^{\prime \prime} \supset S^{\prime} \supset S_{5}$ satisfying the inequality $\left|\mathrm{Fn} S^{\prime \prime}\right|>\left|\mathrm{Fn} S^{\prime}\right|+\delta\left(S^{\prime}\right)$ is well generated over $S_{5}$.

Proof of Lemma 6.4(1)-(3) For the computation, we need to show that Step 3 of the algorithm applies, i.e., that any sufficiently large $\mathfrak{P}_{5}$-geometric extension of any configuration $S_{5}$ is well generated. If rk $S_{5}=18$, we apply Lemma A.7, checking the hypotheses case by case. If $D=5$, there are four configurations $S_{5}$ of rank 17, and Lemma A.7 implies that any sufficiently large extension $S^{\prime \prime} \supset S_{5}$ of rank 19 is well generated. A further extension $S^{\prime \prime \prime} \supset S^{\prime \prime}$ is well generated whenever

$$
\left|\mathrm{Fn} S^{\prime \prime \prime}\right|>\left|\mathrm{Fn} S^{\prime \prime}\right|+4
$$

the worst case scenario being that of $S^{\prime \prime}$ having but two disjoint generating lines. The computation shows that $\left|\mathrm{Fn} S^{\prime \prime}\right| \leqslant 25$ and, if $\left|\mathrm{Fn} S^{\prime \prime}\right|=25$, the pencil $\mathcal{P}_{S^{\prime \prime}}(\Sigma)$ has a type $\mathbf{A}_{2}$ fiber with both lines generating, reducing (A.4) to $\left|\mathrm{Fn} S^{\prime \prime \prime}\right|>27$. It follows that any $\mathfrak{P}_{5}$-geometric extension $S \supset S_{5}$ with $|\mathrm{Fn} S|>28$ is well generated, and Step 3 of the algorithm results in a unique such extension, viz. $\Phi_{30}^{\prime \prime \prime}$.

\section{A.8 Astral Configurations}

Let $\Sigma \cong \widetilde{\mathbf{D}}_{4}$ and $\Gamma:=\mathcal{P}(\Sigma)$. The central line of a type $\widetilde{\mathbf{D}}_{4}$ fiber $\Sigma^{\prime} \subset \Gamma$ is the only line $l \in \Sigma^{\prime}$ of relative valency 4 . The following simple observations follow from the assumption that girth $(\Gamma) \geqslant 6$ :

- all sections of $\Gamma$ are simple or double (i.e., bisections); 
- a section $s \in \sec \Gamma$ is double if and only if it intersects the central line of any (equivalently, each) type $\widetilde{\mathbf{D}}_{4}$ fiber;

- if $\Gamma$ has a bisection, then it has no fibers of type $\widetilde{\mathbf{A}}_{5}$;

- if $\Gamma$ has two bisections, then it has no parabolic fibers other than $\Sigma$.

Proof of Lemma 5.2 We proceed as in Sect. A.5.4, mainly using Lemma A.3. Below, we consider extensions of a configuration $S_{5}$ spanned by $h$ and $\Sigma \cup \sec \Gamma$.

Each of the three sets sec $\Gamma$ of cardinality 12 is covered by Lemma A.3: one has rk $S_{5}=18$ and any $\mathfrak{P}_{5}$-geometric extension $S^{\prime \prime} \supset S_{5}$ such that $\left|\mathrm{Fn} S^{\prime \prime}\right|>22$ is well generated. Using Sect. A.5.4, we immediately obtain Statement (1).

There are several sets of cardinality 11 , but we only consider the three that are geometric for $D=5$. Two of these sets have more than one bisection; hence, $\Gamma$ has no parabolic fibers other than $\Sigma$. We have rk $S_{5}=16$ and the computation shows that each well-generated geometric extension $S^{\prime \prime} \supset S^{\prime} \supset S_{5}$ of corank $c=2$ or 1 has exactly $c$ extra lines. Since all fibers of $\Gamma$ other than $\Sigma$ are of types $\mathbf{A}_{p}, p \leqslant 4$, each generating line can intersect at most two other lines $l \in \Gamma$. Hence, any further extension $S \supset S_{5}$ such that $|\mathrm{Fn} S|>\left|\mathrm{Fn} S_{5}\right|+2+4=22$ must be well generated; using Sect. A.5.4, we show that such extensions do not exist.

In the third case, there is a single bisection $s$ and $\mathrm{rk} S_{5}=17$. Well-generated geometric extensions $S^{\prime \prime} \supset S^{\prime} \supset S_{5}$ of corank $c=2$ or 1 have up to $c+1$ extra lines, which are all generating, pairwise disjoint, and disjoint from $s$, implying that an extra line $e$ cannot be the central line of a type $\widetilde{\mathbf{D}}_{4}$ fiber of $\Gamma$; hence, as in the previous case, $e$ intersects at most two other lines $l \in \Gamma$. As above, we conclude that any geometric extension $S \supset S_{5}$ such that $|\mathrm{Fn} S|>21$ must be well generated; the classification of such extensions using Sect. A.5.4 proves that $|\mathrm{Fn} S| \leqslant 21$.

Acknowledgements I am grateful to Sławomir Rams and Ichiro Shimada for fruitful and motivating discussions. My special gratitude goes to Dmitrii Pasechnik for his indispensable help in "identifying" some of the Fano graphs. This paper was revised and finalized during my stay at the Abdus Salam International Centre for Theoretical Physics, Trieste; I would like to thank this institution and its friendly staff for their warm hospitality and excellent working conditions.

\section{References}

1. Bourbaki, N.: Lie Groups and Lie Algebras. Chapters 4-6. Elements of Mathematics (Berlin). Springer, Berlin (2002). Translated from the 1968 French original by Andrew Pressley

2. Degtyarev, A.: Lines in supersingular quartics (2016). arXiv:1604.05836

3. Degtyarev, A.: Smooth models of singular K3-surfaces (2016). arXiv:1608.06746

4. Degtyarev, A.: Large Fano graphs (2017). http://www.fen.bilkent.edu.tr/ degt/papers/Fano_graphs. zip

5. Degtyarev, A., Itenberg, I., Sertöz, A.S.: Lines on quartic surfaces. Math. Ann. 368(1-2), 753-809 (2017)

6. Dolgachev, I.V.: Classical Algebraic Geometry. Cambridge University Press, Cambridge (2012)

7. GAP-Groups, Algorithms, and Programming, Version 4.7 .7 (2015). http://www.gap-system.org

8. Kulikov, Vik. S.: Surjectivity of the period mapping for $K 3$ surfaces. Usp. Mat. Nauk. 32(4), 257-258 (1977) (in Russian)

9. Nikulin, V.V.: Integer symmetric bilinear forms and some of their geometric applications. Math. USSR Izv. 14(1), 103-167 (1980)

10. Nikulin, V.V.: Weil linear systems on singular $K 3$ surfaces. In: Fujiki, A. (ed.) Algebraic Geometry and Analytic Geometry. ICM-90 Satellite Conference Proceedings, pp. 138-164. Springer, Tokyo (1991) 
11. Pjateckiı̌-Šapiro, I.I., Šafarevič, I.R.: A Torelli theorem for algebraic surfaces of type K3. Math. USSR Izv. 5(3), 547-588 (1971)

12. Rams, S., Schütt, M.: 64 Lines on smooth quartic surfaces. Math. Ann. 362(1-2), 679-698 (2015)

13. Reid, M.: Hyperelliptic linear systems on a K3 surface. J. Lond. Math. Soc. 13(3), 427-437 (1976)

14. Saint-Donat, B.: Projective models of $K-3$ surfaces. Am. J. Math. 96, 602-639 (1974)

15. Schur, F.: Ueber eine besondre Classe von Flächen vierter Ordnung. Math. Ann. 20(2), 254-296 (1882)

16. Segre, B.: The maximum number of lines lying on a quartic surface. Q. J. Math., Oxf. Ser. 14, 86-96 (1943)

17. Shimada, I., Shioda, T.: On a smooth quartic surface containing 56 lines which is isomorphic as a $K 3$ surface to the Fermat quartic. Manuscr. Math. 153(1-2), 279-297 (2017) 